\title{
Sums of two relatively prime cubes
}

by

\author{
R. C. Baker (Provo, UT)
}

1. Introduction. Let $V(x)$ be the number of solutions $(u, v)$ in $\mathbb{Z}^{2}$ of

$$
|u|^{3}+|v|^{3} \leq x, \quad(u, v)=1,
$$

and let

$$
E(x)=V(x)-\frac{4 \Gamma^{2}(1 / 3)}{\pi^{2} \Gamma(2 / 3)} x^{2 / 3}
$$

be the error term in the asymptotic formula for $V(x)$.

Recent progress in estimating $E(x)$ has been conditional on the Riemann hypothesis (R.H.). It is known that, for any $\varepsilon>0$,

$$
E(x)=O\left(x^{331 / 1254+\varepsilon}\right)
$$

if R.H. holds (Zhai and Cao [26]). Earlier bounds are due to Moroz [15], Nowak [19], Müller and Nowak [16], Nowak [17-19] and Zhai [25]. I shall prove

THEOREM 1. We have, subject to R.H.,

$$
E(x)=O\left(x^{\theta+\varepsilon}\right)
$$

where $\theta=9581 / 36864$.

For comparison,

$$
331 / 1254=0.26395 \ldots, \quad 9581 / 36864=0.25990 \ldots
$$

The correct exponent in this problem is likely to be $2 / 9$ (see for example, Zhai [25]), which would make (1.2) an improvement of over $9 \%$ on (1.1).

In the first instance, the improvement depends on a decomposition of sums

$$
\sum_{D<n \leq D^{\prime}} \mu(n) f(n)
$$

2000 Mathematics Subject Classification: Primary 11P21; Secondary 11L07, 11N25.

Key words and phrases: sums of two relatively prime cubes, Riemann hypothesis, bilinear exponential sums. 
into "Type I" and "Type II" sums. The decomposition is a slight variant of that of Heath-Brown [7] for sums

$$
\sum_{D<n \leq D^{\prime}} \Lambda(n) f(n) .
$$

Here the complex function $f$ is arbitrary, and $1<D<D^{\prime} \leq 2 D$. The decomposition is more flexible than that of Montgomery and Vaughan [14], which is used in $[25,26]$. See $\S 2$ for details.

The second component of the method is a collection of exponential sum estimates in two integer variables, which we assemble in $\S \S 3-5$. It is helpful to compare these, and the way they are applied, with [25, 26]. The proof of Theorem 1 reduces to the upper estimation of the quantities $E_{1}(x), E_{2}(x)$ introduced in $\S 6$. Theorem 2 is used to dispatch $E_{2}(x)$. When I wrote the first version of this paper, this was a substantial improvement (based on [21]) of the treatment in Zhai [25]. While the first version was being refereed, I found that Zhai and Cao [26] had given a similar treatment of $E_{2}(x)$. Clearly, then, the present paper is stronger than [26] through the treatment of $E_{1}(x)$. Zhai and Cao use only one method to estimate Type II sums

$$
S(M, N)=\sum_{\substack{m \sim M \\ D<m n \leq D^{\prime}}} \sum_{\substack{n \sim N \\ D<m}} a_{m} e\left(\frac{x^{1 / 3}}{m n}\right),
$$

namely Theorem 2 of [1], a "three variable" method. Since one of the variables reduces to the value 1 , a refinement of the theorem is possible (Theorem 6 below). I deploy three further estimates for Type II sums (Lemmas 5, 7 and Theorem 5).

When it comes to Type I sums $\left(b_{n} \equiv 1\right.$ in $\left.S(M, N)\right)$, Zhai and Cao treat the variable $m$ trivially. I supplement this with Theorems 4 and 8 . Moreover, the decomposition of (1.3) in [26] requires the Type II method to work for

$$
N \in\left[D^{1 / 3}, D^{1 / 2}\right]
$$

and the Type I method only for the "easy" range $N>D^{2 / 3}$. In contrast, for a particular range of $D$ in the relevant interval $\left[x^{0.13 \cdots}, x^{0.22 \cdots}\right]$, I examine what ranges of $N$ are accessible for Types I and II, and then choose a "decomposition result" from $\S 2$ to take advantage of this information.

I now comment briefly on Theorems 3, 4, 5, 7 and 8. The approach in Theorems 3-5 resembles [3], but the outcome is different because in [3] a "degeneracy" occurs. Theorem 7 is essentially a generalization of $[6$, Theorem 6.12] while Theorem 8 is an application of Theorem 7 to Type I sums.

At one point in [6], there is an implicit use of a relation

$$
u \frac{\partial^{2} f_{1}}{\partial u^{2}} \asymp \frac{\partial f_{1}}{\partial u}
$$


which I could not verify (see the appeal to Lemma 6.10 for $T_{0}$ on page 84 ). The proof of Theorem 6 bypasses this difficulty. The argument also allows for another lacuna in [6]: the proof will not work unless $R=\sqrt{Z Y / X} \geq 1$. Hence, in optimizing the estimate

$$
S^{2} \ll N^{2} Z^{-1}+F N^{1 / 12} Z^{1 / 2}+\cdots+F^{1 / 2} N^{1 / 4} Y Z^{3 / 4}
$$

on page 85, extra terms $F N^{1 / 2} X^{1 / 2} Y^{-1 / 2}, F^{1 / 2} N^{1 / 4} Y^{1 / 4} X^{3 / 4}$ must appear. (It should be emphasized that [6] is much clearer than any discussion of similar two-dimensional sums elsewhere in the literature.)

In $\S 6$, I recapitulate from the literature a decomposition

$$
E(x)=E_{1}(x)+E_{2}(x)+E_{3}(x)
$$

and use R.H. to dispatch $E_{3}(x)$, essentially as in [25]. The treatment of $E_{2}(x)$ is also contained in $\S 6$. In $\S 7$, I complete the proof of Theorem 1 with the treatment of $E_{1}(x)$.

We conclude this section with a few remarks on notation. We assume, as we may, that $\varepsilon$ is sufficiently small. In later sections, real constants $\alpha, \beta, \gamma$ appear.

The symbol $c$ is reserved for a sufficiently small positive constant depending at most on $\alpha, \beta, \gamma$. Constants implied by " $O$ " and " $\ll$ " notations depend at most on $\alpha, \beta, \gamma$ and also (in $\S \S 1,2,3,6,7$ ) on $\varepsilon$. We write $A \asymp B$ if

$$
A \ll B \ll A \text {. }
$$

The cardinality of a finite set $E$ is denoted by $|E|$. The symbol $D$ always denotes a large positive number, and $D^{\prime}$ satisfies $D<D^{\prime} \leq 2 D$. We write " $n \sim N$ " as an abbreviation for " $N<n \leq 2 N$ ". We reserve the symbols $I, J$ for bounded real intervals.

2. Decomposition of sums involving the Möbius function. Let

$$
Y=(2 D)^{1 / k},
$$

where $k$ is a natural number, $k \leq \varepsilon^{-1}$. Let

$$
M(s)=\sum_{n \leq Y} \mu(n) n^{-s} .
$$

It is easy to verify the identity

$$
\frac{1}{\zeta(s)}=\sum_{j=1}^{k}(-1)^{j-1}\left(\begin{array}{c}
k \\
j
\end{array}\right) \zeta(s)^{j-1} M(s)^{j}+\zeta(s)^{-1}(1-\zeta(s) M(s))^{k} .
$$

This is nearly the same as (6) of [7], which we can recover from (2.1) by multiplying by $\zeta^{\prime}(s)$. 
Since

$$
\sum_{n=1}^{\infty} \frac{\mu(n)}{n^{s}}=\frac{1}{\zeta(s)} \quad(\operatorname{Re} s>1)
$$

we can express the coefficient of $f(n)$ in the sum

$$
S(f)=\sum_{D<n \leq D^{\prime}} \mu(n) f(n)
$$

by picking out the coefficient of $n^{-s}$ on the right in (2.1). The last term makes no contribution, since

$$
1-\zeta(s) M(s)=\sum_{n>Y} a(n) n^{-s}
$$

for suitable $a(n)$. On splitting up the ranges of summation into ranges $(N, 2 N](N \geq 1 / 2)$, we find that $S(f)$ is a linear combination of $O\left((\log D)^{2 k-1}\right)$ sums of the form

$$
\sum_{n_{i} \sim N_{i}, D<n_{1} \ldots n_{2 k-1} \leq D^{\prime}} \mu\left(n_{k}\right) \ldots \mu\left(n_{2 k-1}\right) f\left(n_{1} \ldots n_{2 k-1}\right),
$$

where $\prod_{i=1}^{2 k-1} N_{i} \asymp D$ and

$$
2 N_{i} \leq Y \quad \text { if } i \geq k \text {. }
$$

We may allow one or more of the $N_{i}$ to be $1 / 2$, so that $n_{i}=1$. This explains why $k$ is the same in (2.1), (2.3).

We now define a Type I sum to be a sum of the form

$$
S_{1}=S_{1}(M, N)=\sum_{\substack{m \sim M \\ D<m n \leq D^{\prime}}} a_{m} \sum_{\substack{n \sim N \\ D<m}} f(m n)
$$

in which $a_{m} \ll m^{\varepsilon}$ for every $\varepsilon>0$. A Type $I I$ sum is a sum of the form

$$
S_{2}=S_{2}(M, N)=\sum_{\substack{m \sim M \\ D<m n \leq D^{\prime}}} \sum_{\substack{n \sim N \\ D<m}} a_{m} f(m n)
$$

in which $a_{m} \ll m^{\varepsilon}, b_{n} \ll n^{\varepsilon}$ for every $\varepsilon>0$.

Lemma 1. Let $0 \leq \alpha_{1} \leq \cdots \leq \alpha_{r}, \alpha_{1}+\cdots+\alpha_{r}=1$. For $S \subseteq\{1, \ldots, r\}$, we write $S^{\prime}=\{1, \ldots, r\} \backslash S$ and

$$
\sigma_{S}=\sum_{i \in S} \alpha_{i}
$$

(i) Let $h$ be an integer, $h \geq 3$. Suppose that $\alpha_{r} \leq 2 /(h+1)$. Then some $\sigma_{S} \in[1 / h, 2 /(h+1)]$.

(ii) Let $\lambda \geq 2 / 3$ and suppose that $\alpha_{r} \leq \lambda$. Then some $\sigma_{S} \in[1-\lambda, 1 / 2]$.

(iii) Let $\varrho \in(1 / 3,2 / 5]$ and $\tau=\min (1-2 \varrho, 3 / 10)$. Suppose that $\alpha_{r} \leq \varrho$. Then some $\sigma_{S} \in[\tau, 1 / 3] \cup[2 / 5,1 / 2]$. 
(iv) Let $\chi \leq 1 / 5$ and

$$
\psi \geq \max (1 / 3,1 / 5+4 \chi / 5) .
$$

Suppose that $\alpha_{r} \leq 2 \chi$. Then some $\sigma_{S} \in[\chi, \psi]$.

Proof. In each case, we suppose that the conclusion is false and obtain a contradiction.

(i) Let $T$ be the set of $i$ for which $\alpha_{i} \in[0,2 /(h+1)-1 / h]$. Then $\sigma_{T}<$ $1 / h$, for otherwise the least $\sigma_{S}$ with $S \subseteq T, \sigma_{S} \geq 1 / h$ would have

$$
\sigma_{S} \leq \frac{1}{h}+\left(\frac{2}{h+1}-\frac{1}{h}\right)=\frac{2}{h+1} .
$$

Our next step is to show that $\left|T^{\prime}\right|=h$. If $\left|T^{\prime}\right|<h$, then

$$
1=\sigma_{T}+\sigma_{T^{\prime}}<\left|T^{\prime}\right| h^{-1}+h^{-1} \leq 1,
$$

which is absurd. So $\left|T^{\prime}\right| \geq h$.

Let $i, i^{\prime}$ be distinct elements of $T^{\prime}$. Then

$$
\alpha_{i}+\alpha_{i^{\prime}} \geq 2\left(\frac{2}{h+1}-\frac{1}{h}\right) \geq \frac{1}{h} .
$$

Consequently, $\alpha_{i}+\alpha_{i^{\prime}}>2 /(h+1)$. It follows that

$$
\sigma_{T^{\prime}}>\frac{\left|T^{\prime}\right|}{2} \frac{2}{h+1}=\frac{\left|T^{\prime}\right|}{h+1} .
$$

Clearly $\left|T^{\prime}\right|=h$. Now (2.7) yields

$$
\sigma_{T}<\frac{1}{h+1} .
$$

We can improve this bound further. Let $\alpha_{i}=\min _{j \in T^{\prime}} \alpha_{j}$. Then

$$
h \alpha_{i}+\sigma_{T} \leq \sigma_{T^{\prime}}+\sigma_{T}=1 .
$$

Adding on the inequality

$$
(h-1) \sigma_{T}<\frac{h-1}{h+1}
$$

we obtain

$$
h \alpha_{i}+h \sigma_{T}<1+\frac{h-1}{h+1}=\frac{2 h}{h+1} .
$$

Of course it follows that $\alpha_{i}+\sigma_{T}<1 / h$. Now

$$
\sigma_{T}<\frac{1}{h}-\alpha_{i}<\frac{1}{h}-\left(\frac{2}{h+1}-\frac{1}{h}\right)=\frac{2}{h}-\frac{2}{h+1} .
$$

Now let $\alpha_{u}=\max _{j \in T^{\prime}} \alpha_{j}$. From (2.8),

$$
\alpha_{u}+\sigma_{T}<\frac{1}{h}+\left(\frac{2}{h}-\frac{2}{h+1}\right) \leq \frac{2}{h+1},
$$


and it follows that $\alpha_{u}+\sigma_{T}<1 / h$. But now $\sigma_{T^{\prime}}+\sigma_{T} \leq h \alpha_{u}+\sigma_{T}<1$, which is absurd.

(ii) It is clear at once from complementation that no $\sigma_{S} \in[1-\lambda, \lambda]$. Hence $\alpha_{r} \leq 1-\lambda \leq 1 / 3$. From part (i), some $\sigma_{S} \in[1 / 3,1 / 2]$, which is absurd.

(iii) Let $T$ be the set of all $i$ for which $\alpha_{i} \in[0, \tau)$. Then $\sigma_{T}<2 / 5$. To see this, we prove in succession that $\sigma_{S}<2 / 5$ for $S \subseteq T,|S|=2,3, \ldots$. For $|S|=2$, we have $\sigma_{S} \leq 2 \tau \leq 3 / 5$. From the hypothesis, it is clear that $\sigma_{S} \notin[2 / 5,3 / 5]$. So $\sigma_{S}<2 / 5$.

Suppose $\sigma_{S}<2 / 5$ whenever $S \subseteq T,|S|=j$ (where $j \geq 2$ ). For $S \subseteq T$, $|S|=j+1$, then

$$
\sigma_{S}<\frac{j+1}{j} \frac{2}{5} \leq \frac{3}{5},
$$

hence $\sigma_{S}<2 / 5$. This proves our claim that $\sigma_{T}<2 / 5$.

We now have $\sigma_{T^{\prime}}>3 / 5$ and also

$$
1 / 3<\alpha_{i} \leq \varrho \quad\left(i \in T^{\prime}\right) .
$$

It follows that $\left|T^{\prime}\right|=2$. Hence $2 / 3<\sigma_{T^{\prime}} \leq 2 \varrho$, and so $1-2 \varrho \leq \sigma_{T}<1 / 3$. This is absurd.

(iv) Let $T$ be the set of $i$ for which $\alpha_{i} \leq \psi-\chi$. Arguing as in (i) yields $\sigma_{T}<\chi$. Let $U=\left\{i: \alpha_{i} \in(\psi-\chi, \chi)\right\}, V=\left\{i: \alpha_{i} \in(\psi, 2 \chi]\right\}$. Then

$$
\sigma_{U}+\sigma_{V}=1-\sigma_{T}>1-\chi
$$

We cannot have $|V| \geq 2$, for if $|V| \geq 2$, pick distinct $i, j$ in $V$ and let $W=\{i, j\}^{\prime}$; then $\chi \leq 1-4 \chi \leq \sigma_{W}<1-2 \psi \leq \psi$, which is absurd.

Suppose that $|V|=1, V=\{i\}$. Then

$$
\sigma_{U}>1-\chi-\sigma_{V} \geq 1-3 \chi \geq 2 \chi
$$

Hence $|U| \geq 3$. Pick distinct $j, k$ in $U$ and let $W=\{i, j, k\}$. Then, since $\psi \geq 1 / 4+\chi / 2$ (as we easily verify), we have

$$
1-\psi \leq 3 \psi-2 \chi<\sigma_{W} \leq 4 \chi \leq 1-\chi, \quad \chi \leq \sigma_{W^{\prime}}<\psi
$$

This is absurd, so $V$ is empty. Now $\sigma_{U}>1-\chi \geq 4 \chi$. So $|U| \geq 5$. Pick distinct $i, j, k, l$ in $U$ and let $W=\{i, j, k, l\}$. Then

$$
1-\psi \leq 4 \psi-4 \chi<\sigma_{W}<4 \chi \leq 1-\chi,
$$

leading to a contradiction once more.

We use this combinatorial lemma in conjunction with the familiar notion of grouping variables in (2.3).

Lemma 2. Let $h, \lambda, \varrho, \tau, \chi, \psi$ be as in Lemma 1 . Let $B>0$ and let $f$ be a complex function on $\mathbb{Z} \cap\left(D, D^{\prime}\right]$. 
(i) Suppose that every Type I sum with

$$
N \gg D^{2 /(h+1)}
$$

satisfies

$$
S_{1}(M, N) \ll B
$$

and every Type II sum with

$$
D^{1 / h} \ll N \ll D^{2 /(h+1)}
$$

satisfies

$$
S_{2}(M, N) \ll B .
$$

Then

$$
S(f) \ll B(\log 3 D)^{A}
$$

with $A=2 h-1$.

(ii) Suppose that every Type I sum with

$$
M \gg D^{\lambda}
$$

satisfies (2.9), and every Type II sum with

$$
D^{1-\lambda} \ll M \ll D^{1 / 2}
$$

satisfies (2.10). Then (2.11) holds with $A=3$.

(iii) Suppose that every Type I sum with

$$
M \gg D^{\varrho}
$$

satisfies (2.9), and every Type II sum with

$$
D^{\tau} \ll M \ll D^{1 / 3} \quad \text { or } \quad D^{2 / 5} \ll M \ll D^{1 / 2}
$$

satisfies (2.10). Then (2.11) holds with $A=5$.

(iv) Suppose that every Type I sum with

$$
M \gg D^{2 \chi}
$$

satisfies (2.9), and every Type II sum with

$$
D^{\chi} \ll M \ll D^{\psi}
$$

satisfies (2.10). Then (2.11) holds with $A=5$.

Proof. (i) Take $k=h$ in (2.3), so that $1 / k<2 /(h+1)$. We must show that every sum $(2.3)$ is $\ll B$. If some $N_{i}>\varepsilon D^{2 /(h+1)}$, we must have $i<k$ from (2.4). Now we group the variables in (2.3) as

$$
n=n_{i}, \quad m=\prod_{\substack{j=1 \\ j \neq i}}^{2 k-1} n_{j}
$$

and appeal to (2.9). 
Now suppose that

$$
N_{j} \leq \varepsilon D^{2 /(h+1)} \quad(1 \leq i \leq 2 k-1) .
$$

Let $D_{0}=2^{2 k-1} N_{1} \ldots N_{2 k-1}$ and write

$$
2 N_{i}=D_{0}^{\alpha_{i}} \text {. }
$$

Then $\alpha_{i} \geq 0, \alpha_{1}+\cdots+\alpha_{2 k-1}=1, D \ll D_{0} \ll D$, and each $\alpha_{i} \leq 2 /(h+1)$. By Lemma $1(\mathrm{i})$, we have $\sigma_{S} \in[1 / h, 2 /(h+1)]$ for some $S \subseteq T$. Clearly

$$
D^{1 / h} \ll N:=\prod_{i \in S} N_{i} \ll D^{2 /(h+1)} .
$$

Thus we may group the variables in such a way that the sum (2.3) becomes a linear combination of $O(1)$ Type II sums satisfying (2.10). The desired estimate follows at once.

(ii) Take $k=2$ in $(2.3)$, so that $1 / k<\lambda$. The argument is very similar to the proof of (i), with $N_{i} \leq \varepsilon D^{\lambda}(1 \leq i \leq 3)$ in place of $(2.12)$, and with $D^{1-\lambda} \ll M=\prod_{i \in S} N_{i} \ll D^{1 / 2}$ in place of $(2.13)$.

(iii), (iv) Take $k=3$ in (2.3). The argument follows the same lines as above, and we can omit the details.

We conclude this section by recording an elementary lemma that will be used for "optimizations" in $\S \S 3-5$.

Lemma 3 ([6, Lemma 2.4]). Let $t \ll 1, u \ll 1$, and

$$
L(H)=\sum_{i=1}^{t} A_{i} H^{a_{i}}+\sum_{j=1}^{u} B_{j} H^{-b_{j}}
$$

where $A_{i}, B_{j}, a_{i}, b_{j}$ are positive. Let $0<H_{1} \leq H_{2}$. Then there is some $H \in\left[H_{1}, H_{2}\right]$ with

$$
L(H) \ll \sum_{i=1}^{t} \sum_{j=1}^{u}\left(A_{i}^{b_{j}} B_{j}^{a_{i}}\right)^{1 /\left(a_{i}+b_{j}\right)}+\sum_{i=1}^{t} A_{i} H_{1}^{a_{i}}+\sum_{j=1}^{u} B_{j} H_{2}^{-b_{j}} .
$$

\section{Estimates for exponential sums}

Lemma 4. Let $\beta$ be a real constant, $\beta \leq 4, \beta(\beta-1) \neq 0$. Let $M>1 / 2$, $\delta>0$. Let $\mathcal{N}(M, \delta)$ denote the number of integer quadruples $\left(m_{1}, m_{2}, \widetilde{m}_{1}, \widetilde{m}_{2}\right)$, $1 \leq m_{i} \leq M, 1 \leq \widetilde{m}_{i} \leq M$, such that

$$
\left|m_{1}^{\beta}+m_{2}^{\beta}-\widetilde{m}_{1}^{\beta}-\widetilde{m}_{2}^{\beta}\right| \leq \delta M^{\beta} \text {. }
$$

Then

$$
\mathcal{N}(M, \delta) \ll M^{2+\varepsilon}+\delta M^{4+\varepsilon} .
$$

Proof. Robert and Sargos ([21, Theorem 2]) give the corresponding result for quadruples satisfying (3.1) and

$$
m_{i} \sim M, \quad \tilde{m}_{i} \sim M \quad(i=1,2) .
$$


(The restriction $\beta \leq 4$ does not occur in their result.) We indicate the details of their argument that have to be changed in order to get Lemma 4 .

Clearly we may suppose that $M$ is a power of 2. By Lemma 1 of [21],

$$
\mathcal{N}(M, \delta) \ll \delta \int_{0}^{\delta^{-1}}\left|\sum_{m=1}^{M} e\left(x\left(\frac{m}{M}\right)^{\beta}\right)\right|^{4} d x .
$$

By a splitting-up argument combined with Minkowski's inequality, there is an interval $I$ of the form $(H, 2 H], H \leq M$, or [1,2], such that

$$
\mathcal{N}(M, \delta) \ll L^{4} \delta \int_{0}^{\delta^{-1}}\left|\sum_{m \in I} e\left(x\left(\frac{m}{M}\right)^{\beta}\right)\right|^{4} d x .
$$

Here $L=\log (M+2)$. If $I=[1,2]$, then trivially $\mathcal{N}(M, \delta) \ll L^{4} \delta \delta^{-1}=L^{4}$. So we may suppose that $I=(H, 2 H]$.

Make a change of variable $y=x\left(H M^{-1}\right)^{\beta}$ in the above integral. We obtain

$$
\mathcal{N}(M, \delta) \ll L^{4} \delta\left(H M^{-1}\right)^{-\beta} \int_{0}^{\delta^{-1}\left(H M^{-1}\right)^{\beta}}\left|\sum_{m \in I} e\left(y\left(\frac{m}{H}\right)^{\beta}\right)\right|^{4} d y .
$$

If the upper limit of integration satisfies $\delta^{-1}\left(H M^{-1}\right)^{\beta} \leq H^{2}$, then it follows from $[21$, Lemma 7] that

$$
\mathcal{N}(M, \delta) \ll L^{4} \delta\left(H M^{-1}\right)^{-\beta} H^{4+\varepsilon / 2} \ll L^{4} \delta M^{4+\varepsilon / 2} \ll \delta M^{4+\varepsilon},
$$

since $\beta \leq 4$.

Suppose now that $\delta^{-1}\left(H M^{-1}\right)^{\beta}>H^{2}$. Then

$$
\begin{aligned}
& \int_{0}^{\delta^{-1}\left(H M^{-1}\right)^{\beta}}\left|\sum_{m \in I} e\left(y\left(\frac{m}{H}\right)^{\beta}\right)\right|^{4} d y \\
& \ll L^{4}\left\{\frac{\delta^{-1}\left(H M^{-1}\right)^{\beta}}{H^{2}}\right\} \int_{0}^{H^{2}}\left|\sum_{m \in I} e\left(y\left(\frac{m}{H}\right)^{\beta}\right)\right|^{4} d y \quad([21, \text { Lemma } 3]) \\
& \ll \delta^{-1}\left(H M^{-1}\right)^{\beta} M^{2+\varepsilon / 2}
\end{aligned}
$$

by a further application of [21, Lemma 7]. Combining (3.3), (3.4), we obtain $\mathcal{N}(M, \delta) \ll M^{2+\varepsilon}$. This completes the proof of Lemma 4 .

We introduce the notation

$$
\left|\left(m_{1}, m_{2}\right)\right|_{\alpha}=\left(\left|m_{1}\right|^{\alpha}+\left|m_{2}\right|^{\alpha}\right)^{1 / \alpha}
$$

for an integer pair $\left(m_{1}, m_{2}\right)$. The following theorem is roughly comparable to Lemma 4.2 of [26]. 
TheORem 2. Let $(\kappa, \lambda)$ be an exponent pair. Let $\gamma$, $\beta$ be constants, $\gamma<1$, $\gamma \neq 0,1<\beta \leq 4$. Let $M \geq 1 / 2,1 / 2 \leq M_{1} \leq M_{2}, X \gg M_{2}^{2}$. Let $\left|a_{m}\right| \leq 1$, $\left|b_{m_{1}, m_{2}}\right| \leq 1$, and

$$
S=\sum_{m \sim M} \sum_{m_{1} \sim M_{1}} \sum_{m_{2} \sim M_{2}} a_{m} b_{m_{1}, m_{2}} e\left(\frac{X m^{\gamma}\left|\left(m_{1}, m_{2}\right)\right|_{\beta}}{M^{\gamma} M_{2}}\right) .
$$

Then

$$
S \ll_{\varepsilon} M M_{2}^{1+\varepsilon}+M M_{2}^{2+\varepsilon}\left(\frac{X}{M_{2}^{2}}\right)^{\kappa /(2+2 \kappa)} M^{-(1+\kappa-\lambda) /(2+2 \kappa)} .
$$

We remark that if $M_{1}=M_{2}$, Theorem 2 is of the same strength as the estimate for trilinear sums

$$
\sum_{m \sim M} \sum_{m_{1} \sim M_{1}} \sum_{m_{2} \sim M_{2}} a_{m} b_{m_{1}, m_{2}} e\left(\frac{X m^{\gamma} m_{1}^{\alpha_{1}} m_{2}^{\alpha_{2}}}{M^{\gamma} M_{1}^{\alpha_{1}} M_{2}^{\alpha_{2}}}\right)
$$

obtained by Heath-Brown's method [8]. See for example [1, Theorem 2]. The estimate (3.5) deteriorates for fixed $M_{1}$ and increasing $M_{2}$, but this will not cost us anything in the application in $\S 6$.

Proof of Theorem 2. For $m_{1} \sim M_{1}, m_{2} \sim M_{2}$, we have

$$
\left|\left(m_{1}, m_{2}\right)\right|_{\beta} \in\left[c_{1} M_{2}, c_{2} M_{2}\right]
$$

where $c_{1}, c_{2}$ are suitable positive constants. Let $Q$ be an arbitrary natural number. We divide $\left[c_{1} M_{2}, c_{2} M_{2}\right]$ into intervals $I_{1}, \ldots, I_{Q}$ of equal length, so that

$$
|S| \leq \sum_{m \sim M} \sum_{q=1}^{Q}\left|\sum_{\substack{\left.m_{i} \sim M_{i} \\\left(m_{1}, m_{2}\right)\right|_{\beta} \in I_{q}}} b_{m_{1}, m_{2}} e\left(\frac{X m^{\gamma}\left|\left(m_{1}, m_{2}\right)\right|_{\beta}}{M^{\gamma} M_{2}}\right)\right| .
$$

Cauchy's inequality gives

$$
|S|^{2} \leq M Q \sum_{q=1}^{Q} \sum_{(3.7)}\left|\sum_{m \sim M} e\left(\frac{X m^{\gamma} D(\mathbf{m})}{M^{\gamma} M_{2}}\right)\right|,
$$

where

$$
\mathbf{m}=\left(m_{1}, m_{2}, \widetilde{m}_{1}, \widetilde{m}_{2}\right) \quad\left(m_{j}, \widetilde{m}_{j} \sim M_{j}\right), \quad D(\mathbf{m})=\left|\left(m_{1}, m_{2}\right)\right|_{\beta}-\left|\left(\widetilde{m}_{1}, \widetilde{m}_{2}\right)\right|_{\beta}
$$

and the sum over $\mathbf{m}$ in (3.6) is restricted by

$$
\left|\left(m_{1}, m_{2}\right)\right|_{\beta} \in I_{q}, \quad\left|\left(\widetilde{m}_{1}, \widetilde{m}_{2}\right)\right|_{\beta} \in I_{q} .
$$

Clearly

$$
|S|^{2} \leq M Q \sum_{(3.9)}\left|\sum_{m \sim M} e\left(\frac{X m^{\gamma} D(\mathbf{m})}{M^{\gamma} M_{2}}\right)\right|
$$


where $\mathbf{m}$ is restricted by

$$
|D(\mathbf{m})| \leq\left(c_{2}-c_{1}\right) \frac{M_{2}}{Q}
$$

A splitting-up argument yields

$$
|S|^{2} \ll M Q L \sum_{(3.11)}\left|\sum_{m \sim M} e\left(\frac{X m^{\gamma} D(\mathbf{m})}{M^{\gamma} M_{2}}\right)\right| .
$$

Here $L=\log 3 M_{2}$ and the sum over $\mathbf{m}$ is restricted by

$$
\left(\Delta-\frac{1}{M_{2}^{2}}\right) M_{2} \leq|D(\mathbf{m})|<2 \Delta M_{2} \text {. }
$$

The positive number $\Delta$ is of the form

$$
\Delta=2^{h} M_{2}^{-2}, \quad \Delta \ll Q^{-1}, \quad h \geq 0 .
$$

Now it is easy to see that (3.11) implies (3.1) with $M_{2}$ in place of $M$ and a suitable $\delta \ll \Delta$. Accordingly, the number of quadruples satisfying (3.11) is

$$
\ll M_{2}^{2+\varepsilon}+\Delta M_{2}^{4+\varepsilon} \text {. }
$$

If $h=0$ in (3.12), we use the trivial estimate for the inner sum in (3.10). It follows that

$$
|S|^{2} \ll M^{2} Q L M_{2}^{2+\varepsilon} .
$$

If $h>0$, the number of quadruples satisfying (3.11) is $\ll \Delta M_{2}^{4+\varepsilon}$. By the definition of an exponent pair, we have

$$
\sum_{m \sim M} e\left(\frac{X m^{\gamma} D(\mathbf{m})}{M^{\gamma} M_{2}}\right) \ll(X \Delta)^{\kappa} M^{\lambda-\kappa}+\left(X \Delta M^{-1}\right)^{-1}
$$

for any quadruple $\mathbf{m}$ counted in (3.10). Here the contribution to the righthand side of $(3.10)$ from $\left(X \Delta M^{-1}\right)^{-1}$ is

$$
\ll M Q L \Delta M_{2}^{4+\varepsilon} X^{-1} \Delta^{-1} M \ll M_{2}^{4+2 \varepsilon} M^{2} X^{-1} Q \ll M^{2} Q M_{2}^{2+2 \varepsilon}
$$

since $X \gg M_{2}^{2}$. The remaining contribution is

$$
\ll M Q \Delta M_{2}^{4+2 \varepsilon}(X \Delta)^{\kappa} M^{\lambda-\kappa} \ll M^{1+\lambda-\kappa} Q^{-\kappa} M_{2}^{4+2 \varepsilon} X^{\kappa}
$$

from (3.12). Collecting (3.13)-(3.15) gives

$$
S \ll M M_{2}^{1+\varepsilon} Q^{1 / 2}+M^{(1+\lambda-\kappa) / 2} M_{2}^{2+\varepsilon} X^{\kappa / 2} Q^{-\kappa / 2} .
$$

Now the theorem follows on applying Lemma 3 with $H_{1}=1$ and arbitrarily large $\mathrm{H}_{2}$.

LEMMA 5. Let $\alpha, \beta$ be real constants with $\alpha \beta(\alpha-1)(\beta-1) \neq 0, X>0$, $M \geq 1, N \geq 1,\left|a_{m}\right| \leq 1,\left|b_{n}\right| \leq 1$, and $1<D<D^{\prime}$. Let $L=\log (2+X M N)$. 
Let $(\kappa, \lambda)$ be an exponent pair and

$$
S(M, N)=\sum_{\substack{m \sim M \\ D<m n \leq D^{\prime}}} \sum_{\substack{n \sim N \\ D<m}} a_{m} e\left(\frac{X m^{\alpha} n^{\beta}}{M^{\alpha} N^{\beta}}\right) .
$$

Then

$$
\begin{aligned}
S(M, N) \ll & L^{3}\left\{\left(X^{2+4 \kappa} M^{8+10 \kappa} N^{9+11 \kappa+\lambda}\right)^{1 /(12+16 \kappa)}\right. \\
& +X^{1 / 6} M^{2 / 3} N^{3 / 4+\lambda /(12+12 \kappa)}+\left(X M^{3} N^{4}\right)^{1 / 5} \\
& +\left(X M^{7} N^{10}\right)^{1 / 11}+M^{2 / 3} N^{11 / 12+\lambda /(12+12 \kappa)} \\
& \left.+M N^{1 / 2}+\left(X^{-1} M^{14} N^{23}\right)^{1 / 22}+X^{-1 / 2} M N\right\} .
\end{aligned}
$$

Proof. At the cost of a factor $L$, we can remove the condition $D<m n \leq$ $D^{\prime}$ from the sum in (3.17). See the discussion in the proof of [2, Lemma 11]. Now the result follows at once from Theorem 2 of $\mathrm{Wu}$ [24]. (As pointed out by $\mathrm{Wu}$, his theorem is essentially an abstraction of an idea of Jia [11].)

Lemma 6. Let $\alpha_{j}$ be nonzero constants and $M_{j} \geq 1(1 \leq j \leq 4)$. Let $X>0$ and $\left|a_{m_{1} m_{2}}\right| \leq 1,\left|b_{m_{3} m_{4}}\right| \leq 1$. Let $L=\log 2 M_{1} M_{2} M_{3} M_{4}$. We have

$$
\begin{aligned}
\sum_{\substack{m_{j} \sim M \\
m_{1} m_{2} m_{3} m_{4} \leq D^{\prime}}} a_{m_{1} m_{2}} b_{m_{3} m_{4}} e\left(\frac{X m_{1}^{\alpha_{1}} m_{2}^{\alpha_{2}} m_{3}^{\alpha_{3}} m_{4}^{\alpha_{4}}}{M_{1}^{\alpha_{1}} M_{2}^{\alpha_{2}} M_{3}^{\alpha_{3}} M_{4}^{\alpha_{4}}}\right) \\
\ll L^{2}\left\{\left(X M_{1} M_{2} M_{3} M_{4}\right)^{1 / 2}+M_{1} M_{2}\left(M_{3} M_{4}\right)^{1 / 2}\right. \\
\left.+\left(M_{1} M_{2}\right)^{1 / 2} M_{3} M_{4}+X^{-1 / 2} M_{1} M_{2} M_{3} M_{4}\right\} .
\end{aligned}
$$

Proof. We remove the condition $D<m_{1} m_{2} m_{3} m_{4} \leq D^{\prime}$ as explained in the preceding proof. Now the result follows from Theorem 2 of Fouvry and Iwaniec [5].

The key element of the proof of Theorem 2 of [5] is the double large sieve of Bombieri and Iwaniec [4]. The same applies to the following result of Robert and Sargos [21], but they need the difficult "counting lemma" stated as Lemma 4, above.

Lemma 7. Let $\alpha, \beta, \gamma$ be constants, $\alpha(\alpha-1) \beta \gamma \neq 0$. Let $H, M, N$ be positive integers and $X>1$. Let $\left|a_{h, n}\right| \leq 1,\left|b_{m}\right| \leq 1$. Then

$$
\begin{aligned}
& \sum_{h \sim H} \sum_{\substack{n \sim N \\
D<m n \leq D^{\prime}}} a_{h, n} \sum_{\substack{m \sim M \\
D<}} b_{m}\left(\frac{X h^{\beta} n^{\gamma} m^{\alpha}}{H^{\beta} N^{\gamma} M^{\alpha}}\right) \\
& \ll(H N M)^{1+\varepsilon}\left\{\left(\frac{X}{H N M^{2}}\right)^{1 / 4}+\frac{1}{(H N)^{1 / 4}}+\frac{1}{M^{1 / 2}}+\frac{1}{X^{1 / 2}}\right\} .
\end{aligned}
$$


Proof. After the preliminary removal of the condition $D<m n \leq D^{\prime}$ as above, this reduces to Theorem 1 of [21].

4. Applications of the theory of exponent pairs. We begin with a simple lemma from [12] (Lemma 2.8).

Lemma 8. Let $K>0$ and let $f$ be a continuously differentiable function on $[N, 2 N]$ with

$$
F_{0}=\max _{N \leq t \leq 2 N}|f(t)|, \quad F_{1}=\max _{N \leq t \leq 2 N}\left|f^{\prime}(t)\right|>0 .
$$

Then

$$
\sum_{n \sim N} \min \left\{K, \frac{1}{\|f(n)\|}\right\} \ll\left(F_{0}+1\right)\left(K+F_{1}^{-1} \log 2 N\right) .
$$

We next state a version of the " $B$-process".

Lemma 9. Suppose that $f$ has four continuous derivatives on $[N, 2 N]$, $f^{\prime \prime}>0$ on $[N, 2 N]$, and for some $F>0$,

$$
\left|f^{(j)}(t)\right| \asymp F N^{-j} \quad(t \sim N, 2 \leq j \leq 4) .
$$

Let $t_{\nu}$ be defined by $f^{\prime}\left(t_{\nu}\right)=\nu$, and let $\phi(\nu)=-f\left(t_{\nu}\right)+\nu t_{\nu}$. Let $L=$ $\log \left(F N^{-1}+2\right)$. Then for $[a, b] \subseteq[N, 2 N]$,

$$
\begin{aligned}
\sum_{a \leq n \leq b} e(f(n))= & \sum_{f^{\prime}(a) \leq \nu \leq f^{\prime}(b)} \frac{e(\phi(\nu)+1 / 8)}{f^{\prime \prime}\left(t_{\nu}\right)^{1 / 2}} \\
& +O\left(L+\min \left(F^{-1 / 2} N,\left\|f^{\prime}(a)\right\|^{-1}\right)\right. \\
& \left.+\min \left(F^{-1 / 2} N,\left\|f^{\prime}(b)\right\|^{-1}\right)\right) .
\end{aligned}
$$

Proof. This can easily be obtained by an elaboration of the proof of $[6$, Lemma 3.6] with $-f$ in place of $f$, and $-\nu$ in place of $\nu$. We simply separate the smallest term from the sum

$$
\sum_{H_{1} \leq \nu \leq H_{2}} \min \left(\left|-f^{\prime}(a)+\nu\right|^{-1}, F^{-1 / 2} N\right)
$$

on p. 29, and proceed similarly with $b$ in place of $a$.

We now add to the hypothesis of Lemma 9 the assumption that

$$
f^{(j)}(t)=\left(\begin{array}{c}
\alpha \\
j
\end{array}\right) A t^{\alpha-j}\left(1+R_{j}(t)\right) \quad(0 \leq j \leq 2)
$$

where $\alpha<0, A$ is positive and independent of $t$, and

$$
\left|R_{j}(t)\right|<c^{2} \quad(a \leq t \leq b, 0 \leq j \leq 2) .
$$

Then

$$
|\alpha| A t_{\nu}^{\alpha-1}\left(1+R_{1}\left(t_{\nu}\right)\right)=-\nu>0 .
$$


Hence

$$
t_{\nu}=(|\alpha| A)^{-1 /(\alpha-1)}\left(1+R\left(t_{\nu}\right)\right)(-\nu)^{1 /(\alpha-1)},
$$

with $R(t)=\left(1+R_{1}(t)\right)^{-1 /(\alpha-1)}-1$. So

$$
\begin{aligned}
f^{\prime \prime}\left(t_{\nu}\right) & =\frac{1}{2} \alpha(\alpha-1) A t_{\nu}^{\alpha-2}\left(1+R_{2}\left(t_{\nu}\right)\right) \\
& =\tau A^{1 /(\alpha-1)}(-\nu)^{(\alpha-2) /(\alpha-1)}\left(1+R^{*}\left(t_{\nu}\right)\right)
\end{aligned}
$$

with $\tau=-|\alpha|^{1 /(\alpha-1)}(\alpha-1) / 2, R^{*}(t)=\left(1+R_{2}(t)\right)(1+R(t))^{\alpha-2}-1$.

Similarly,

$$
\begin{aligned}
\phi(\nu) & =-A t_{\nu}^{\alpha}\left(1+R_{0}\left(t_{\nu}\right)\right)-(|\alpha| A)^{-1 /(\alpha-1)}\left(1+R\left(t_{\nu}\right)\right)(-\nu)^{\alpha /(\alpha-1)} \\
& =\gamma A^{-1 /(\alpha-1)}(-\nu)^{\alpha /(\alpha-1)}\left(1+\widehat{R}\left(t_{\nu}\right)\right)
\end{aligned}
$$

with $\gamma=-|\alpha|^{-\alpha /(\alpha-1)}-|\alpha|^{-1 /(\alpha-1)}$ and

$$
\gamma(1+\widehat{R}(t))=-|\alpha|^{-\alpha /(\alpha-1)}\left(1+R_{0}(t)\right)(1+R(t))^{\alpha}-|\alpha|^{-1 /(\alpha-1)}(1+R(t)) .
$$

The point is that

$$
\max \left(\left|R^{*}(t)\right|,|\widehat{R}(t)|\right)<c \quad(a \leq t \leq b) .
$$

We now permit $A$ to depend on a variable $u$ :

$$
A=C g(u) \quad(u \sim M, M \ll N)
$$

where $C>0$,

$$
g(u)=u^{\beta}\left(1+\sum_{j \geq 1} d_{j}(B / u)^{j}\right) \quad(u \sim M)
$$

where $\beta$ is a nonzero constant,

$$
\alpha+\beta<1, \quad 0<B<c M,
$$

and the power series $\sum_{j} d_{j} z^{j}$ converges in the unit disc. Writing $h(t)=$ $t^{\alpha}\left(1+R_{0}(t)\right)$, we have

$$
f(t, u)=C h(t) g(u)
$$

in place of $f(t)$. Let $F=C M^{\beta} N^{\alpha}$. We rewrite (4.1), with $[a(m), b(m)]$ in place of $[a, b]$, as

$$
\begin{aligned}
& \sum_{a(m) \leq n \leq b(m)} e(f(n, m)) \\
= & \sum_{A(m) \leq \nu \leq B(m)} \frac{W(\nu) e(G(\nu, m))}{g(m)^{1 /(2 \alpha-2)}} \\
& +O\left(L+\min \left(F^{-1 / 2} N,\|A(m)\|^{-1}\right)+\min \left(F^{-1 / 2} N,\|B(m)\|^{-1}\right)\right) .
\end{aligned}
$$


Here

$$
\begin{aligned}
W(\nu) & =\left(\tau C^{1 /(\alpha-1)}\left(1+R^{*}\left(t_{\nu}\right)\right)(-\nu)^{(\alpha-2) /(\alpha-1)}\right)^{-1 / 2} e(1 / 8), \\
G(\nu, m) & =\gamma(-\nu)^{\alpha /(\alpha-1)} C^{-1 /(\alpha-1)}\left(1+\widehat{R}\left(t_{\nu}\right)\right) g(m)^{-1 /(\alpha-1)}, \\
A(m) & =C g(m) h^{\prime}(a(m)), \quad B(m)=C g(m) h^{\prime}(b(m)) .
\end{aligned}
$$

We apply this formula to the sum

$$
S(h, g, C)=\sum_{m \sim M} \sum_{a(m) \leq n \leq b(m)} e(C h(n) g(m))
$$

where $[a(m), b(m)] \subset(N, 2 N]$. Summing over $m$ in (4.5) and interchanging summations yields

$$
\begin{aligned}
S(h, g, C)= & \sum_{\nu \ll F N^{-1}} W(\nu) \sum_{m \in E_{\nu}} \frac{e(G(\nu, m))}{g(m)^{1 /(2 \alpha-2)}} \\
& +O\left(M L+\sum_{m \sim M} \min \left(F^{-1 / 2} N, \frac{1}{\|G(m)\|}\right)\right) .
\end{aligned}
$$

Here $G(m)$ is one of $A(m), B(m)$, and

$$
E_{\nu}=\{m \sim M: A(m) \leq \nu \leq B(m)\} .
$$

Let us suppose that

(4.8) $G$ is continuously differentiable and $G^{\prime}(m) \gg F(M N)^{-1}$,

(4.9) $F \gg N$.

In view of Lemma 8 and the monotonicity of $g$, (4.6) yields

$$
\begin{aligned}
S(h, g, C) & \ll\left(F N^{-2}\right)^{-1 / 2} F N^{-1} \max _{\substack{\nu \ll F N^{-1} \\
I \subseteq(M, 2 M]}}\left|\sum_{m \in I} e\left(G_{\nu}(m)\right)\right|+M L+F^{1 / 2} \\
& \ll F^{1 / 2+\kappa} M^{\lambda-\kappa}+M L
\end{aligned}
$$

for any exponent pair $(\kappa, \lambda)$. It is clear that $\partial G(\nu, m) / \partial m^{j}$ satisfies the required conditions $([6$, pp. $30-31])$ for the last bound, for $j=1,2, \ldots$. (The exponent in $m^{-\beta /(\alpha-1)}$ is less than 1 , by hypothesis.)

We summarize our conclusions in the following theorem. In the language of [6], the theorem asserts that $(1 / 2,1 / 2 ; \kappa, \lambda)$ is an exponent quadruple.

Theorem 3. Let $(\kappa, \lambda)$ be an exponent pair. Define $S(h, g, C)$ by (4.6), with the assumptions on $h$ and $g$ made above. Let $F=C M^{\beta} N^{\alpha}, M \ll N$, $L=\log \left(F N^{-1}+2\right)$. Suppose further that (4.7) and (4.8) hold. Then

$$
S(h, g, C) \ll F^{1 / 2+\kappa} M^{\lambda-\kappa}+M L+M N F^{-1} .
$$


Note that the condition (4.9) has been dropped and the term $M N F^{-1}$ incorporated in (4.11). This is justified since, if $F<c N$, the Kuz'minLandau theorem ([6, Theorem 2.1]) gives

$$
S(h, g, C)=\sum_{m \sim M} O\left(F^{-1} N\right) \ll M N F^{-1} .
$$

We now apply Theorem 3 to Type I sums.

Lemma 10. Let $K \geq 1, M \geq 1,\left|u_{k, m}\right| \leq 1(k \sim K, m \sim M)$. Let $I_{m} \subseteq(K, 2 K]$. There is a real number $t$ such that

$$
\sum_{m \sim M}\left|\sum_{k \in I_{m}} u_{k, m}\right| \ll(\log 2 K) \sum_{m \sim M}\left|\sum_{k \sim K} u_{k, m} e(k t)\right| .
$$

Proof. From Lemma 2.2 of [4], there is a positive continuous function $F_{K}(t)$ on $\mathbb{R}$ such that $\int_{\mathbb{R}} F_{K}(t) d t \ll \log 2 K$ and

$$
\left|\sum_{k \in I_{m}} u_{k, m}\right| \leq \int_{-\infty}^{\infty} F_{K}(t)\left|\sum_{k \sim K} u_{k, m} e(k t)\right| d t .
$$

Thus the left-hand side of (4.13) is

$$
\leq \int_{-\infty}^{\infty} F_{K}(t) \sum_{m \sim M}\left|\sum_{k \sim K} u_{k, m} e(k t)\right| d t \ll \log 2 K \max _{t} \sum_{m \sim M}\left|\sum_{k \sim K} u_{k, m} e(k t)\right|,
$$

as required.

TheOREM 4. Let $(\kappa, \lambda)$ be an exponent pair. Let $\alpha, \beta$ be constants, $\alpha \neq 0$, $\alpha<1, \beta<0$. Let $X>0, M \geq 1 / 2, N \geq 1 / 2, M N \asymp D, N_{0}=\min (M, N)$, $L=\log (D+2)$. Let $\left|a_{m}\right| \leq 1, I_{m} \subseteq(N, 2 N]$, and

$$
S_{1}=\sum_{m \sim M} a_{m} \sum_{n \in I_{m}} e\left(\frac{X m^{\beta} n^{\alpha}}{M^{\beta} N^{\alpha}}\right)
$$

Then

$$
S_{1} \ll L^{2}\left\{D N^{-1 / 2}+D X^{-1}+\left(D^{4+4 \kappa} X^{1+2 \kappa} N^{-(1+2 \kappa)} N_{0}^{2(\lambda-\kappa)}\right)^{1 /(6+4 \kappa)}\right\} .
$$

Proof. We may suppose that $N$ is large. If $X<c N$, we proceed as in (4.12). Now suppose that $X \geq c N$. Let $Q$ be a natural number, $Q<c^{2} N$.

By Lemma 10, there is a real number $t$ such that

$$
S_{1} \ll L \sum_{m \sim M}\left|\sum_{n \sim N} e\left(\frac{X m^{\beta} n^{\alpha}}{M^{\beta} N^{\alpha}}+t n\right)\right| .
$$


By the Cauchy-Schwarz inequality and the Weyl-van der Corput inequality $([6,(2.3 .4)])$, and writing $I(q)=(N, 2 N-q]$, we have

$$
\begin{aligned}
S_{1}^{2} & \ll L^{2} M \sum_{m \sim M}\left|\sum_{n \sim N} e\left(\frac{X m^{\beta} n^{\alpha}}{M^{\beta} N^{\alpha}}+t n\right)\right|^{2} \\
& \ll L^{2} D^{2} Q^{-1}+L^{2} D Q^{-1} \sum_{q=1}^{Q} \sum_{m \sim M} \sum_{n \in I(q)} e\left(\frac{X m^{\beta}\left((n+q)^{\alpha}-n^{\alpha}\right)}{M^{\beta} N^{\alpha}}+t q\right) .
\end{aligned}
$$

After applying a splitting-up argument to the sum over $q$, we find that there is a $q \in[1, Q]$ for which

$$
S_{1}^{2} \ll L^{2} D^{2} Q^{-1}+L^{3} D q Q^{-1}\left|\sum_{m \sim M} \sum_{n \in I(q)} e\left(\frac{X m^{\beta}\left((n+q)^{\alpha}-n^{\alpha}\right)}{M^{\beta} N^{\alpha}}\right)\right| .
$$

After a straightforward verification that the conditions are satisfied, we may apply Theorem 3 to the above double sum, with either $\left(n^{\beta},(m+q)^{\alpha}-\right.$ $\left.m^{\alpha}\right)$ or $\left((n+q)^{\alpha}-n^{\alpha}, m^{\beta}\right)$ in the role of $h(n), g(m)$ (depending on whether $N_{0}$ is $N$ or $\left.M\right)$. Thus

$$
\begin{gathered}
F \asymp X N^{-1} q, \\
L^{-4} S_{1}^{2} \ll D^{2} Q^{-1}+D\left(X^{1 / 2+\kappa} N^{-1 / 2-\kappa} N_{0}^{\lambda-\kappa} Q^{1 / 2+\kappa}+N_{0}+D X^{-1} N Q^{-1}\right) .
\end{gathered}
$$

We can drop the last two terms since

$$
D N_{0} \ll D^{2} N^{-1} \ll D^{2} Q^{-1}, \quad D^{2} X^{-1} Q^{-1} N \ll D^{2} Q^{-1} .
$$

The resulting bound for $L^{-4} S_{1}^{2}$ holds, in fact, for $0<Q<c^{2} N$. An application of Lemma 3 completes the proof.

We now adapt this proof to estimate a Type II sum.

THEOREM 5. Make the hypothesis of Theorem 4 and suppose that $\left|b_{n}\right| \leq 1$. Let

$$
S_{2}=\sum_{m \sim M} a_{m} \sum_{\substack{n \sim N \\ D<m n \leq D^{\prime}}} b_{n} e\left(\frac{X m^{\beta} n^{\alpha}}{M^{\beta} N^{\alpha}}\right)
$$

Suppose further that

$$
N \ll M, \quad X \gg D
$$

Then

$$
S_{2} \ll L^{7 / 4}\left(D N^{-1 / 2}+D M^{-1 / 4}+\left(D^{11+10 \kappa} X^{1+2 \kappa} N^{2(\lambda-\kappa)}\right)^{1 /(14+12 \kappa)}\right) .
$$

Proof. We remove the condition $D<m n \leq D^{\prime}$ at the cost of a factor $L$. Let $Q$ be a positive integer, $Q<c^{2} N$. As in the preceding proof, there is a 
$q \in[1, Q]$ such that (writing $I_{m, q}=I_{m} \cap\left(I_{m}-q\right)$ )

$$
\begin{aligned}
L^{-2} S_{2}^{2} & \ll D^{2} Q^{-1}+\frac{D q L}{Q}\left|\sum_{m \sim M} \sum_{n \sim N} \bar{b}_{n} b_{n+q} e\left(\frac{X m^{\beta}\left((n+q)^{\alpha}-n^{\alpha}\right)}{M^{\beta} N^{\alpha}}\right)\right| \\
& \ll D^{2} Q^{-1}+\frac{D q L}{Q} \sum_{n \sim N}\left|\sum_{m \sim M} e\left(\frac{X m^{\beta}\left((n+q)^{\alpha}-n^{\alpha}\right)}{M^{\beta} N^{\alpha}}\right)\right| .
\end{aligned}
$$

Suppose further that $Q<c M^{1 / 2}$. Using the Weyl-van der Corput inequality again, we obtain

$$
\begin{aligned}
& \left|\sum_{m \sim M} e\left(\frac{X m^{\beta}\left((n+q)^{\alpha}-n^{\alpha}\right)}{M^{\beta} N^{\alpha}}\right)\right|^{2} \\
& \quad \leq \frac{M^{2}}{Q^{2}}+\frac{L M q^{\prime}}{Q^{2}} \sum_{m \in J\left(q^{\prime}\right)} e\left(\frac{X\left(\left(m+q^{\prime}\right)^{\beta}-m^{\beta}\right)\left((n+q)^{\alpha}-n^{\alpha}\right)}{M^{\beta} N^{\alpha}}\right)
\end{aligned}
$$

for some $q^{\prime} \leq Q^{2}$, with $J\left(q^{\prime}\right)=\left(M, 2 M-q^{\prime}\right]$. Combining this with (4.18) and Cauchy's inequality, we have

$$
L^{-4} S_{2}^{4} \ll \frac{D^{4} L^{2}}{Q^{2}}+\frac{D^{3} q^{2} q^{\prime} L^{3}}{Q^{4}} S_{M, N}
$$

where

$$
S_{M, N}=\sum_{n \sim N} \sum_{m \in J\left(q^{\prime}\right)} e\left(\frac{X\left(\left(m+q^{\prime}\right)^{\beta}-m^{\beta}\right)\left((n+q)^{\alpha}-n^{\alpha}\right)}{M^{\beta} N^{\alpha}}\right) .
$$

An application of Theorem 3 to $S_{M, N}$ now yields

$$
L^{-7} S_{2}^{4} \ll \frac{D^{4}}{Q^{2}}+D^{3}\left(\left(\frac{X Q^{3}}{D}\right)^{1 / 2+\kappa} N^{\lambda-\kappa}+N+\frac{D^{2}}{X Q^{3}}\right)
$$

(this is of course also true for $0<Q<1$ ).

We may discard the term $D^{5} / X Q^{3}$ since $D^{5} / X Q^{3} \leq D^{5} / X Q^{2} \ll D^{4} / Q^{2}$. An application of Lemma 3 with $H_{1} \rightarrow 0+, H_{2}=\min \left(c^{2} N, c M^{1 / 2}\right)$ yields

$$
L^{-7} S_{2}^{4} \ll D^{4} N^{-2}+D^{4} M^{-1}+\left(D^{11+10 \kappa} X^{1+2 \kappa} N^{2(\lambda-\kappa)}\right)^{2 /(7+6 \kappa)}+D^{3} N .
$$

Since $D^{3} N \ll D^{4} M^{-1}$, the theorem follows at once.

We now pursue a variant of the above arguments.

Theorem 6. Let $(\kappa, \lambda)$ be an exponent pair. Let $\alpha, \beta$ be constants, $\alpha \neq 1$, $\beta<0, \alpha+\beta<2$. Let $N \geq 1 / 2, X \gg N, M \gg N, M N \asymp D, L=$ $\log (X D+2)$. Let $\left|a_{m}\right| \leq 1,\left|b_{n}\right| \leq 1$. Let

$$
S_{2}=\sum_{\substack{m \sim M \\ D<m n \leq D^{\prime}}} a_{m} \sum_{\substack{n \sim N \\ D<}} b_{n} e\left(\frac{X m^{\beta} n^{\alpha}}{M^{\beta} N^{\alpha}}\right) .
$$


Then

$$
S_{2} \ll L^{2}\left(D N^{-1 / 2}+D M^{-1 / 4}+X^{1 / 6}\left(D^{4+5 \kappa} N^{\lambda-\kappa}\right)^{1 /(6+6 \kappa)}\right) .
$$

Proof. Let $Q$ be a positive integer, $Q<c^{2} \min \left(N, M^{1 / 2}\right)$. As in (4.18), there is a $q, 1 \leq q \leq Q$, for which

$$
L^{-2} S_{2}^{2} \ll D^{2} Q^{-1}+\frac{D q L}{Q} \sum_{n \sim N}\left|\sum_{m \sim M} e\left(\frac{X m^{\beta}\left((n+q)^{\alpha}-n^{\alpha}\right)}{M^{\beta} N^{\alpha}}\right)\right| .
$$

We apply (4.5) to the inner sum, with the roles of $n, m$ reversed, so that $(\alpha, \beta)$ is replaced by $(\beta, \alpha-1)$ :

$$
\sum_{m \sim M} e\left(\frac{X m^{\beta}\left((n+q)^{\alpha}-n^{\alpha}\right)}{M^{\beta} N^{\alpha}}\right)=\sum_{\nu \in I_{n}} \frac{W(\nu)}{g(n)^{1 /(2 \beta-2)}} e(G(\nu, n))
$$

$+O\left(L+\min \left(\left(\frac{X q}{N}\right)^{-1 / 2},\|A(n)\|^{-1}\right)+\min \left(\left(\frac{X q}{N}\right)^{-1 / 2},\|B(n)\|^{-1}\right)+\frac{D}{X q}\right)$.

Here

$$
B(n)=\frac{X}{M^{\beta} N^{\alpha}}\left((n+q)^{\alpha}-n^{\alpha}\right) \beta M^{\beta-1}, \quad A(n)=2^{\beta-1} B(n),
$$

and $I_{n}=[A(n), B(n)]$. The last term on the right-hand side of (4.20) allows for a possible application of the Kuz'min-Landau inequality.

Combining (4.19), (4.20) shows that there are numbers $w_{\nu},\left|w_{\nu}\right| \leq 1$, with

$$
\begin{aligned}
L^{-2} S_{2}^{2} \ll & D^{2} Q^{-1}+\frac{L q D}{Q}\left(\frac{X q}{D M}\right)^{-1 / 2} \sum_{n}\left|\sum_{\nu \in I_{n}} w_{\nu} e(G(\nu, n))\right| \\
& +L^{2} D N+L D X^{1 / 2} Q^{1 / 2} N^{-1 / 2}
\end{aligned}
$$

Since $D N \ll D^{2} Q^{-1}$ from $Q<N \ll D^{1 / 2}$, we have

$$
\begin{aligned}
L^{-4} S_{2}^{2} \ll & D^{2} Q^{-1}+D X^{1 / 2} Q^{1 / 2} N^{-1 / 2} \\
& +Q^{-1 / 2} X^{-1 / 2} D^{3 / 2} M^{1 / 2} \sum_{n}\left|\sum_{\nu \in I_{n}} w_{\nu} e(G(\nu, n))\right| .
\end{aligned}
$$

We apply the Cauchy and Weyl-van der Corput inequalities to obtain

$$
\begin{aligned}
L^{-8} S_{2}^{4} & \ll D^{4} Q^{-2}+D^{2} X Q N^{-1} \\
& +(Q X)^{-1} D^{4}\left(\frac{(X q / D)^{2}}{H}+\frac{X q}{D}\left|\sum_{n} \sum_{\nu \in I_{n} \cap\left(I_{n}-h\right)} e\left(G_{1}(\nu, n)\right)\right|\right)
\end{aligned}
$$

with $H$ (specified below) satisfying $0<H<c X q / D$ and some $h, 1 \leq h \leq H$. Here

$$
G_{1}(\nu, n)=G(\nu+h, n)-G(\nu, n) .
$$


We make the obvious choice $H=Q q^{2} N X / D^{2}$; the assumption $Q<c^{2} M^{1 / 2}$ yields $H<c(X q / D)$.

We interchange the summations over $n$ and $\nu$ in (4.21). Once $\nu$ is fixed, $n$ runs over a single interval. We apply the method of exponent pairs to $\sum_{n} e\left(G_{1}(\nu, n)\right)$; the order of size of $G_{1}$ is

$$
\frac{h}{X q / D} \frac{X q}{N} \asymp h M \gg N
$$

Thus

$$
\sum_{n} e\left(G_{1}(\nu, n)\right) \ll(h M)^{\kappa} N^{\lambda-\kappa} \ll\left(Q^{3} X / D\right)^{\kappa} N^{\lambda-\kappa} .
$$

Combining this with (4.21) gives

$$
\begin{aligned}
L^{-8} S_{2}^{4} & \ll D^{4} Q^{-2}+D^{2} X Q N^{-1}+D^{2} X Q\left(Q^{3} X / D\right)^{\kappa} N^{\lambda-\kappa} \\
& \ll D^{4} Q^{-2}+D^{2} X Q N^{-1}+D^{2-\kappa} X^{1+\kappa} Q^{1+3 \kappa} N^{\lambda-\kappa} .
\end{aligned}
$$

An application of Lemma 3 yields

$$
\begin{aligned}
L^{-8} S_{2}^{4} \ll & D^{4 / 3}\left(D^{2} X N^{-1}\right)^{2 / 3}+\left(D^{4}\right)^{(1+3 \kappa) /(3+3 \kappa)}\left(D^{2-\kappa} X^{1+\kappa} N^{\lambda-\kappa}\right)^{2 /(3+3 \kappa)} \\
& +D^{4} M^{-1}+D^{4} N^{-2} \\
\ll & D^{4 / 3} X^{2 / 3} N^{-2 / 3}+X^{2 / 3}\left(D^{8+10 \kappa} N^{\lambda-\kappa}\right)^{2 /(3+3 \kappa)}+D^{4} M^{-1}+D^{4} N^{-2} .
\end{aligned}
$$

In the last expression, the first term is clearly dominated by the second, and Theorem 6 follows.

5. The AB theorem. Let $X \geq 1, Y \geq 1, N=X Y$. Let $\mathbf{D}$ be a subset of $\mathbf{R}=[X, 2 X] \times[Y, 2 Y]$ satisfying some mild restrictions discussed below. Let $\alpha, \beta$ be real with

$$
(\alpha)_{3}(\beta)_{3}(\alpha+\beta+1)_{2} \neq 0,
$$

where $(\alpha)_{0}=1,(\alpha)_{s}=(\alpha+s-1)(\alpha)_{s-1}$ for $s=1,2, \ldots$

Theorem 6.12 of [6] states that, for $F>0, L=\log (F N+2)$,

$$
\begin{aligned}
S_{f} & :=\sum_{(m, n) \in \mathbf{D}} e\left(F X^{\alpha} Y^{\beta} m^{-\alpha} n^{-\beta}\right) \\
& \ll F^{1 / 3} N^{1 / 2}+N^{5 / 6} L^{2 / 3}+F^{-1 / 8} N^{15 / 16} L^{3 / 8}+F^{-1 / 4} N L^{1 / 2}
\end{aligned}
$$

(the "AB theorem"). In the present section, I extend this by replacing $u^{-\alpha}$, $v^{-\beta}$ by more general functions $h_{1}(u), h_{2}(v)$, with

$$
\begin{array}{cc}
\left(h_{1}(u)-u^{-\alpha}\right)^{(j)} \ll \eta\left|\left(u^{-\alpha}\right)^{(j)}\right| & (u \sim X), \\
\left(h_{2}(v)-v^{-\beta}\right)^{(j)} \ll \eta\left|\left(v^{-\beta}\right)^{(j)}\right| & (v \sim Y),
\end{array}
$$

where $\eta$ is a sufficiently small positive quantity (in terms of $\alpha, \beta$ ) and $j=$ $0,1, \ldots, j \ll 1$. We write $f_{0}(u, v)=A h_{1}(u) h_{2}(v)$ for $(u, v) \in \mathbf{R}$. 
We must deal with some monotonicity conditions for

$$
f_{1}(u, v ; q, r)=f_{0}(u+q, v+r)-f_{0}(u, v)=\int_{0}^{1} \frac{\partial}{\partial t} f_{0}(u+q t, v+r t) d t .
$$

These are a technical nuisance rather than a serious obstacle. We shall see that $\mathbf{R}$ can be partitioned into $O(1)$ rectangles $\mathbf{R}^{\prime}=I \times J$ such that one of $f_{1}^{(2,0)}$ or $f_{1}^{(0,2)}$ has no zero in each $\overline{\mathbf{R}^{\prime}}$. This allows us to impose helpful conditions in Lemmas 11-14 below. In these lemmas, let $f(u, v)$ be a real function on $\bar{I} \times \bar{J}$ and $\mathbf{D} \subseteq \bar{I} \times \bar{J}$. We write $f^{(a, b)}$ for $\partial^{a+b} f / \partial u^{a} \partial v^{b}$. Suppose that $f^{(2,0)}$ is nonzero on $\mathbf{R}$. Let $\psi(w, v)$ denote the solution of

$$
f^{(1,0)}(\psi(w, v), v)=w .
$$

For any function $\phi$ having second order partial derivatives on $\mathbf{D}$, let $H \phi=$ $\phi^{(2,0)} \phi^{(0,2)}-\left\{\phi^{(1,1)}\right\}^{2}$. We need to state some "omega conditions" on $f$, which we assume to be true for the duration of these lemmas.

$\left(\Omega_{1}\right) \quad f$ has partial derivatives of all orders. For a suitable $F>0$ there is a constant $C_{1}$ such that

$$
\left|f^{(a, b)}(u, v)\right| \leq C_{1} F X^{-a} Y^{-b} \quad((u, v) \in \bar{I} \times \bar{J}, 0 \leq a, b \leq 4) .
$$

$\left(\Omega_{2}\right) \quad$ There is a constant $C_{2}$ such that the set

$$
U(v)=\{u:(u, v) \in \mathbf{D}\}
$$

is the union of at most $C_{2}$ intervals for each $v$.

$\left(\Omega_{3}\right) \quad$ There is a constant $C_{3}$ such that the set

$$
V(l)=\{v:(\psi(l, v), v) \in \mathbf{D}\}
$$

is the union of at most $C_{3}$ intervals for each $l$.

A function $f: I \rightarrow \mathbb{R}$ is said to be $C$-monotonic if $I$ can be partitioned into $C$ intervals on each of which $f$ is monotonic.

$\left(\Omega_{4}\right) \quad$ There is a constant $C_{4}$ such that, for each fixed $l, f^{(2,0)}(\psi(l, v), v)$ is $C_{4}$-monotonic on $\bar{J}$.

In Lemmas 11-14, implied constants depend at most on $C_{1}, \ldots, C_{4}$. In Theorem 7, implied constants depend at most on $C_{1}, \ldots, C_{4}, \alpha$ and $\beta$.

Lemma 11 ([6, Lemma 6.6]). Suppose that $\left|f^{(2,0)}\right| \asymp \Lambda$ on $\mathbf{D}$. Then

$$
S_{f} \ll|\mathbf{D}| \Lambda^{1 / 2}+\Lambda^{-1 / 2} Y .
$$

We shall write

$$
g(w, v)=f(\psi(w, v), v)-w \psi(w, v) .
$$


LEMma 12 ([6, proof of Lemma 6.7]). We have

$$
\begin{gathered}
\frac{\partial \psi}{\partial v}(w, v)=-f^{(1,1)}(\psi(w, v), v) / f^{(2,0)}(\psi(w, v), v), \\
\frac{\partial g}{\partial v}=f^{(0,1)}(\psi(w, v), v), \quad \frac{\partial^{2} g}{\partial v^{2}}=\frac{(H f)(\psi(w, v), v)}{f^{(2,0)}(\psi(w, v), v)} .
\end{gathered}
$$

Lemma 13. Suppose that

$$
\left|f^{(j, 0)}\right| \asymp F X^{-j}, \quad\left|f^{(0, j)}\right| \asymp F Y^{-j} \quad(j=1,2), \quad|H f| \asymp F^{2} N^{-2}
$$

and that $\left(\Omega_{2}\right)-\left(\Omega_{4}\right)$ hold, and remain valid with the roles of the variables interchanged. Then

$$
S_{f} \ll F+F^{-1 / 2} N L .
$$

Proof. We may follow the proof of [6, Lemma 6.11] almost verbatim.

We now give a variant of [6], Lemma 6.8.

Lemma 14. Suppose that $\left|f^{(2,0)}\right| \asymp \Lambda$ and $|H f| \asymp M$ on $\mathbf{D}$. Then

$$
S_{f} \ll|\mathbf{D}| M^{1 / 2}+F M^{-1 / 2} X^{-1}+M^{-1 / 2}+Y \Lambda^{-1 / 2}+Y L .
$$

Proof. In view of Lemma 11, we may suppose that $\Lambda \geq M$. Replacing $f$ by $-f$ if necessary, we may suppose that $f^{(2,0)}<0$ on D. Lemma 3.6 of $[6]$ gives

$$
\begin{aligned}
S_{f} & =\sum_{n \sim Y} \sum_{m \in I(n)} e(f(m, n)) \\
& =\sum_{n \sim Y} \sum_{k \in K(n)} e\left(-\frac{1}{8}+g(k, n)\right)\left|f^{(2,0)}(\psi(k, n), n)\right|^{-1 / 2}+O\left(Y \Lambda^{-1 / 2}+Y L\right) .
\end{aligned}
$$

Here $K(n)=\{k:(\psi(k, n), n) \in \mathbf{D}\}$. Changing the order of summation yields

$$
S_{f}=\sum_{k \ll F X^{-1}} \sum_{n \in V(k)} e\left(-\frac{1}{8}+g(k, n)\right)\left|f^{(2,0)}(\psi(k, n), n)\right|^{-1 / 2}+O\left(Y \Lambda^{-1 / 2}+Y L\right) .
$$

Thanks to $\left(\Omega_{3}\right),\left(\Omega_{4}\right)$, we may now apply partial summation to conclude that

$$
S_{f} \ll \sum_{k \ll F X^{-1}} \Lambda^{-1 / 2}\left|\sum_{n \in J(k)} e(g(k, n))\right|+O\left(Y \Lambda^{-1 / 2}+Y L\right)
$$

for an interval $J(k) \subset V(k)$. Now an application of [6, Theorem 2.2], in conjunction with (5.5) and Lemma 12, gives

$$
S_{f} \ll \sum_{k \ll F X^{-1}} \Lambda^{-1 / 2}\left\{\frac{M^{1 / 2}}{\Lambda^{1 / 2}}|V(k)|+\left(\frac{M}{\Lambda}\right)^{-1 / 2}\right\}+Y \Lambda^{-1 / 2}+Y L
$$


Moreover,

$$
\sum_{k}|V(k)|=\sum_{\substack{n \\ \psi(k, n) \in U(n)}} \sum_{\substack{k \\(n,}}
$$

Now the inner sum is the number of integer values assumed by $f^{(1,0)}(m, n)$ as $m$ runs over $U(n)$. Recalling $\left(\Omega_{2}\right)$, the inner sum is

$$
\ll \int_{U(n)} f^{(2,0)}(t, n) d t+1 \ll|U(n)| \Lambda+1 .
$$

Hence

$$
\sum_{k}|V(k)| \ll \sum_{n \sim Y}(|U(n)| \Lambda+1) \ll|\mathbf{D}| \Lambda+Y .
$$

Combining (5.6) and (5.7) gives

$$
S \ll|\mathbf{D}| M^{1 / 2}+\Lambda^{-1} M^{1 / 2} Y+M^{-1 / 2}\left(F X^{-1}+1\right)+Y \Lambda^{-1 / 2}+Y L .
$$

Since $Y \Lambda^{-1 / 2} \geq \Lambda^{-1} M^{1 / 2} Y$, the lemma follows.

For convenience, we record three more lemmas from [6].

LEMMA 15. Let $P$ be a polynomial over $\mathbb{C}$ having distinct zeros, with $P(0) \neq 0$. Let $\delta>0$. Let $q, r$ be integers, $r \neq 0$. Let

$$
E=\left\{(m, n): m \sim X, n \sim Y,\left|P\left(\frac{q n}{r m}\right)\right|<\delta\right\} .
$$

Then

$$
|E|<C(P)\{\delta N+1\} .
$$

Proof. For $q \neq 0$, this follows from [6, Lemma 6.4]. For $q=0, E$ is empty if $\delta$ is sufficiently small, and otherwise the result is trivial.

Lemma 16. Let $P, Q$ be polynomials over $\mathbb{C}$ having no common zero. Let $q, r, m, n$ be integers, $r m \neq 0$. Then

$$
\max \left(\left|P\left(\frac{q n}{r m}\right)\right|,\left|Q\left(\frac{q n}{r m}\right)\right|\right)>C(P, Q)>0 .
$$

Proof. This follows from [6, Lemma 6.5].

LEMma 17 ([6, p. 76]). For $1 \leq Q \leq X, 1 \leq R \leq Y$, we have

$$
S_{f}^{2} \ll \frac{N}{Q R} \sum_{|q| \leq Q} \sum_{|r| \leq R} \sum_{(m, n) \in \mathbf{D}(q, r)} e\left(f_{1}(m, n ; q, r)\right) .
$$

Here $\mathbf{D}(q, r)=\mathbf{D} \cap(\mathbf{D}-(q, r))$ and

$$
f_{1}(m, n ; q, r)=f(m+q, n+r)-f(m, n) .
$$

In Theorem 7 , we write $f_{0} \in \mathcal{E}$ as an abbreviation for the following hypothesis. If $f_{0}$ is restricted to a rectangle $I \times J$ with the property that 
$f_{1}^{(2,0)} \neq 0$ in $E=\bar{I} \times \bar{J}$, then $\left(\Omega_{2}\right),\left(\Omega_{3}\right),\left(\Omega_{4}\right)$ hold for $f_{1}$ when the domain of $f_{1}$ is $\mathbf{D}(q, r, \theta)$ or $\mathbf{D}^{\prime}(q, r, \theta)$ (for all $(q, r) \in \mathbb{Z}^{2}, \theta>0$ ). Here

$$
\begin{aligned}
\mathbf{D}(q, r) & =\{(u, v) \in(E \cap \mathbf{D}) \cap(E \cap \mathbf{D}-(q, r))\}, \\
\mathbf{D}(q, r, \theta) & =\left\{(u, v) \in \mathbf{D}(q, r): \theta \leq\left|H f_{1}\right|<2 \theta\right\}, \\
\mathbf{D}^{\prime}(q, r, \theta) & =\left\{(u, v) \in \mathbf{D}(q, r):\left|H f_{1}\right|<\theta\right\} .
\end{aligned}
$$

Moreover, $f_{0}$ has the same property when the variables interchange roles.

Theorem 7. Let $0<\eta<c(\alpha, \beta)$ where $c$ is sufficiently small. Let $f_{0}$ satisfy (5.2), (5.3) and suppose that $f_{0} \in \mathcal{E}$. Let $F=A X^{-\alpha} Y^{-\beta} \gg N^{1 / 6}$ and $Y \leq X$. Then

$$
\begin{aligned}
S_{f_{0}} \ll & L\left\{F^{1 / 3} N^{1 / 2}+N^{5 / 6}+F^{-1 / 8} N^{15 / 16}+F^{1 / 2} N^{1 / 2} Y^{-1 / 2}\right. \\
& +F^{1 / 12} N^{1 / 2} Y^{5 / 12}+\eta^{2 / 5} N^{1 / 2} F^{1 / 5} Y^{2 / 5} \\
& \left.+\eta^{1 / 4} N^{3 / 4} Y^{1 / 4}+\eta^{1 / 2} F^{1 / 4} N^{1 / 2} Y^{1 / 4}\right\}
\end{aligned}
$$

Proof. We may assume that $F>N^{5 / 6}$ and $Y>N^{1 / 4}$. For suppose that $N^{1 / 6} \ll F \leq N^{5 / 6}$. Since

$$
(-\alpha)(-\alpha-1)(-\beta)(-\beta-1)-\alpha^{2} \beta^{2}=\alpha \beta(\alpha+\beta+1),
$$

it is easy to deduce from (5.2), (5.3) that $H f_{0} \asymp F^{2} N^{-2}$. The omega conditions are rather straightforward to check for $f_{0}$. Hence Lemma 13 gives

$$
S_{f_{0}} \ll F+F^{-1 / 2} N L \ll N^{5 / 6}+F^{-1 / 8} N^{15 / 16} L,
$$

as required. Now suppose that $F>N^{5 / 6}$ and $Y \leq N^{1 / 4}$. We note that (5.9) is trivial for $F>N^{3 / 2}$, so we suppose that $F \leq N^{3 / 2}$. Then Lemma 11 gives

$$
\begin{aligned}
S_{f_{0}} & \ll N\left(F X^{-2}\right)^{1 / 2}+\left(F X^{-2}\right)^{-1 / 2} Y \ll F^{1 / 2} Y+F^{-1 / 8} N^{15 / 16} \\
& \ll F^{1 / 3} N^{1 / 2}+F^{-1 / 8} N^{15 / 16},
\end{aligned}
$$

since $F^{1 / 6} Y N^{-1 / 2} \ll Y N^{-1 / 4} \ll 1$.

We write $f_{1}(u, v)$ rather than $f_{1}(u, v ; q, r)$ for the function in (5.8) with $f=f_{0}$. Let $S=S_{f_{0}}$. From Lemma 17 ,

$$
S^{2} \ll \frac{N^{2}}{Z}+\frac{N}{Z} \sum_{\substack{|q| \leq Q \\(q, r) \neq(0,0)}} \sum_{|r| \leq R} S(q, r)
$$

where $S(q, r)=\sum_{(m, n) \in \mathbf{D}(q, r)} e\left(f_{1}(m, n)\right)$. Here $Z$ is at our disposal subject to $X / Y \leq Z \leq c^{2} N$, and we choose

$$
Q=\sqrt{Z X / Y}, \quad R=\sqrt{Z Y / X}
$$

Note that $Q / X=R / Y=\sqrt{Z / N} \leq c$.

For a fixed pair $q, r$,

$$
\varrho:=\max (|q| / X,|r| / Y) \leq c .
$$


We consider the contribution to the right-hand side of (5.10) from terms with $\varrho=|r| / Y$ (in particular, $r \neq 0$ ). The remaining terms can be estimated similarly.

The hypotheses of the theorem imply that, for bounded $a, b$,

$$
f_{1}^{(a, b)}(m, n)=(-1)^{a+b+1} A m^{-\alpha-a} n^{-\beta-b} \frac{r}{n}\left\{T_{a, b}\left(\frac{q n}{r m}\right)+O(\varrho+\eta)\right\}
$$

where

$$
T_{a, b}(z)=(\alpha)_{a+1}(\beta)_{b} z+(\alpha)_{a}(\beta)_{b+1} .
$$

Moreover,

$$
H f_{1}(m, n)=A^{2} m^{-2 \alpha-2} n^{-2 \beta-2} \frac{r^{2}}{n^{2}}\left\{U\left(\frac{q n}{r m}\right)+O(\varrho+\eta)\right\}
$$

where

$$
U(z)=\alpha \beta(\alpha+\beta+2)\left\{(\alpha)_{2} z^{2}+2(\alpha+1)(\beta+1) z+(\beta)_{2}\right\} .
$$

As pointed out on p. 84 of $[6], U(z)$ has degree 2 and has distinct zeros. We also need the observation that no two of $T_{0,2}, T_{1,1}$ and $T_{2,0}$ have a common zero; nor does $T_{0,2}$ or $T_{2,0}$ share a zero with $U$.

Because of these observations, it suffices to prove (5.9) with $\mathbf{D}$ replaced by a domain $\mathbf{D} \cap(I \times J)$ with the property that $f_{1}^{(2,0)} \neq 0$ in $\bar{I} \times \bar{J}$ or $f_{1}^{(0,2)} \neq 0$ in $\bar{I} \times \bar{J}$. Let us suppose, say, that $f_{1}^{(2,0)} \neq 0$ in $\bar{I} \times \bar{J}$.

Let $\delta$ be a small positive number, to be chosen later. Consider the domains (possibly empty)

$$
\begin{aligned}
\mathbf{D}_{0, j} & =\left\{(m, n) \in \mathbf{D}(q, r): 2^{j} \delta \varrho^{2} F^{2} N^{-2} \leq\left|H f_{1}\right|<2^{j+1} \delta \varrho^{2} F^{2} N^{-2}\right. \\
\text { and } \left.\left|T_{2,0}\left(\frac{q n}{r m}\right)\right| \geq c\right\} \quad(j \geq 0), & \\
\mathbf{D}_{1} & =\left\{(m, n) \in \mathbf{D}(q, r):\left|T_{2,0}\left(\frac{q n}{r m}\right)\right|<c\right\}, \\
\mathbf{D}_{2} & =\left\{(m, n) \in \mathbf{D}(q, r):\left|H f_{1}\right|<\delta \varrho^{2} F^{2} N^{-2}\right\}
\end{aligned}
$$

and set

$$
\begin{gathered}
S_{0, j}=\sum_{(m, n) \in \mathbf{D}_{0, j}} e(f(m, n)), \\
S_{1}=\sum_{(m, n) \in \mathbf{D}_{1}} e(f(m, n)), \quad S_{2}=\sum_{(m, n) \in \mathbf{D}_{2}} e(f(m, n)) .
\end{gathered}
$$

By Lemma 16, the sets $\mathbf{D}_{0, j}(0 \leq j \ll L), \mathbf{D}_{1}, \mathbf{D}_{2}$ form a partition of $\mathbf{D}(q, r)$. Clearly

$$
\left|H f_{1}\right| \asymp 2^{j} \delta \varrho^{2} F^{2} N^{-2} \quad \text { and } \quad\left|f_{1}^{(2,0)}\right| \asymp \varrho F^{2} X^{-2} \quad \text { on } \mathbf{D}_{0, j} \text {. }
$$


Moreover, from Lemma 16,

$$
\begin{gathered}
\left|H f_{1}\right| \asymp \varrho^{2} F^{2} N^{-2} \quad \text { and } \quad\left|f_{1}^{(0,2)}\right| \asymp \varrho F X^{-2} \text { on } \mathbf{D}_{1}, \\
\left|f_{1}^{(2,0)}\right| \asymp \varrho F X^{-2} \quad \text { on } \mathbf{D}_{2} .
\end{gathered}
$$

We may estimate the terms on the right in the decomposition

$$
S(q, r)=\sum_{0 \leq j \ll L} S_{0, j}+S_{1}+S_{2}
$$

by applying Lemmas 11 and 14 . For $\mathbf{D}_{0, j}, \mathbf{D}_{1}, \mathbf{D}_{2}$ are domains $\mathbf{D}(q, r, \theta)$ or $\mathbf{D}^{\prime}(q, r, \theta)$. From Lemma 14 and (5.12),

$$
\begin{aligned}
S_{0, j} \ll & N\left(\varrho^{2} F^{2} N^{-2}\right)^{1 / 2}+\varrho F\left(\delta \varrho^{2} F^{2} N^{-2}\right)^{-1 / 2} X^{-1} \\
& +\left(\delta \varrho^{2} F^{2} N^{-2}\right)^{-1 / 2}+Y\left(\varrho F X^{-2}\right)^{-1 / 2}+Y L \\
\ll & \varrho F+\varrho^{-1 / 2} F^{-1 / 2} N+\delta^{-1 / 2} Y L+\delta^{-1 / 2} \varrho^{-1} F^{-1} N .
\end{aligned}
$$

Similarly, Lemma 14 and (5.13) give

$$
\begin{aligned}
S_{1} \ll & N\left(\varrho^{2} F^{2} N^{-2}\right)^{1 / 2}+\varrho F\left(\varrho^{2} F^{2} N^{-2}\right)^{-1 / 2} Y^{-1} \\
& +\left(\varrho^{2} F^{2} N^{-2}\right)^{-1 / 2}+X\left(\varrho F Y^{-2}\right)^{-1 / 2}+X L \\
\ll & \varrho F+\varrho^{-1} F^{-1} N+X L+\varrho^{-1 / 2} F^{-1 / 2} N .
\end{aligned}
$$

By Lemma 15, and since $\varrho N \geq N / X$, the number of points in $\mathbf{D}_{2}$ is $\ll$ $(\delta+\varrho+\eta) N$. From Lemma 11 and (5.14),

$$
S_{2} \ll(\delta+\varrho+\eta) \varrho^{1 / 2} F^{1 / 2} Y+\varrho^{-1 / 2} F^{-1 / 2} N .
$$

Collecting (5.15)-(5.17) yields

$$
\begin{aligned}
S(q, r) \ll & L \varrho F+L \varrho^{-1 / 2} F^{-1 / 2} N+L^{2} \delta^{-1 / 2} Y \\
& +L \delta^{-1 / 2} \varrho^{-1} F^{-1} N+L N Y^{-1}+(\delta+\varrho+\eta) \varrho^{1 / 2} F^{1 / 2} Y .
\end{aligned}
$$

Note that, since $F \geq N^{5 / 6}$, we have $\varrho F \gg F X^{-1} \gg 1$. We may take $\delta=c(\varrho F)^{-1 / 3}$ to obtain

$$
\begin{aligned}
L^{-2} S(q, r) \ll & \varrho F+\varrho^{-1 / 2} F^{-1 / 2} N+\varrho^{1 / 6} F^{1 / 6} Y \\
& +\varrho^{-5 / 6} F^{-5 / 6} N+N Y^{-1}+\varrho^{3 / 2} F^{1 / 2} Y+\eta \varrho^{1 / 2} F^{1 / 2} Y .
\end{aligned}
$$

Now if $a$ is a constant, $a>-1$, we have

$$
\frac{1}{Z} \sum_{(q, r) \in \mathbf{Q}} \varrho^{a} \ll \frac{1}{Z} \sum_{q=1}^{Q} \sum_{r=1}^{R}\left(\frac{q^{a}}{X^{a}}+\frac{r^{a}}{Y^{a}}\right) \ll\left(\frac{Z}{N}\right)^{a / 2}
$$

where $\mathbf{Q}=\{(q, r):|q| \leq Q,|r| \leq R,(q, r) \neq(0,0)\}$. We combine this with 
(5.10), (5.18) to obtain

$$
\begin{aligned}
L^{-2} S^{2} \ll & N^{2} Z^{-1}+F N^{1 / 2} Z^{1 / 2}+F^{-1 / 2} N^{9 / 4} Z^{-1 / 4} \\
& +F^{1 / 6} N^{11 / 12} Y Z^{1 / 12}+F^{-5 / 6} N^{29 / 12} Z^{-5 / 12}+N^{2} Y^{-1} \\
& +F^{1 / 2} N^{1 / 4} Y Z^{3 / 4}+\eta F^{1 / 2} N^{3 / 4} Y Z^{1 / 4} .
\end{aligned}
$$

We may discard $F^{-5 / 6} N^{29 / 12} Z^{-5 / 12}$ since $F \gg N^{5 / 6}$ :

$$
\begin{aligned}
F^{-5 / 6} N^{29 / 12} Z^{-5 / 12} & =\left(N^{2} Z^{-1}\right)^{11 / 18}\left(F N^{1 / 2} Z^{1 / 2}\right)^{7 / 18} N F^{-11 / 9} \\
& \ll\left(N^{2} Z^{-1}\right)^{11 / 18}\left(F N^{1 / 2} Z^{1 / 2}\right)^{7 / 18} .
\end{aligned}
$$

Applying Lemma 3, we find that

$$
\begin{aligned}
L^{-2} S^{2} \ll & F^{2 / 3} N+F^{2 / 13} N Y^{12 / 13}+F^{2 / 7} N Y^{4 / 7} \\
& +\eta^{4 / 5} N F^{2 / 5} Y^{4 / 5}+N^{5 / 3}+N^{5 / 4} Y^{3 / 4}+F^{-1 / 4} N^{7 / 4} Y^{1 / 4} \\
& +\eta^{1 / 2} N^{3 / 2} Y^{1 / 2}+F N^{1 / 2} X^{1 / 2} Y^{-1 / 2}+F^{1 / 6} N^{11 / 12} X^{1 / 12} Y^{11 / 12} \\
& +F^{1 / 2} N^{1 / 4} X^{3 / 4} Y^{1 / 4}+F^{-1 / 2} N^{2}+N^{2} Y^{-1}+\eta F^{1 / 2} N^{3 / 4} X^{1 / 4} Y^{3 / 4}
\end{aligned}
$$

Clearly we may suppose that $F \ll N^{3 / 2}$. We use $N^{5 / 6} \ll F \ll N^{3 / 2}$, $N^{1 / 4} \ll Y \ll N^{1 / 2}$ to obtain

$$
\begin{aligned}
& F^{-1 / 4} N^{7 / 4} Y^{1 / 4} \ll F^{-1 / 4} N^{15 / 8}, \quad F^{-1 / 2} N^{2} \ll F^{-1 / 4} N^{15 / 8}, \\
& F^{1 / 2} N^{1 / 4} X^{3 / 4} Y^{1 / 4} \ll N X^{3 / 4} Y^{1 / 4} \ll N^{5 / 3}, \quad N^{5 / 4} Y^{3 / 4} \ll N^{5 / 3} .
\end{aligned}
$$

Moreover,

$$
\begin{aligned}
& F^{2 / 7} N Y^{4 / 7} \ll F^{2 / 7} N^{9 / 7} \leq\left(F^{2 / 3} N\right)^{33 / 49}\left(F^{-1 / 2} N^{2}\right)^{16 / 49} \\
& F^{2 / 13} N Y^{12 / 13} \ll F^{2 / 13} N^{19 / 13} \leq\left(F^{2 / 3} N\right)^{63 / 143}\left(F^{-1 / 4} N^{15 / 8}\right)^{80 / 143} .
\end{aligned}
$$

Hence

$$
\begin{aligned}
& L^{-2} S^{2} \ll F^{2 / 3} N+F^{-1 / 4} N^{15 / 8}+\eta^{4 / 5} N F^{2 / 5} Y^{4 / 5}+N^{5 / 3} \\
& +\eta^{1 / 2} N^{3 / 2} Y^{1 / 2}+N^{2} Y^{-1}+\eta F^{1 / 2} N Y^{1 / 2}+F N Y^{-1}+F^{1 / 6} N Y^{5 / 6}
\end{aligned}
$$

We noted above that

$$
S \ll F^{1 / 2} Y+N F^{-1 / 2}, \quad S^{2} \ll F Y^{2}+N^{2} F^{-1} .
$$

If $N^{2} Y^{-1}$ is the maximum term in $(5.20)$

$$
\begin{aligned}
S^{2} & \ll\left(N^{2} Y^{-1}\right)^{2 / 3}\left(F Y^{2}\right)^{1 / 3}+N^{2} F^{-1} \ll N^{4 / 3} F^{1 / 3}+F^{-1 / 4} N^{15 / 8} \\
& \ll\left(F^{2 / 3} N\right)^{1 / 2}\left(N^{5 / 3}\right)^{1 / 2}+F^{-1 / 4} N^{15 / 8},
\end{aligned}
$$

which yields (5.9). We conclude that (5.9) always holds.

Let us now specialize $h_{1}$ and $h_{2}$ for application to Type I sums. We suppose that 
(a) Either

$$
h_{1}(u)=u^{-\alpha} \quad \text { or } \quad h_{1}(u)=\frac{u^{1-\alpha}-(u+p)^{1-\alpha}}{(1-\alpha) p},
$$

where $p>0$ and $p / X$ is sufficiently small.

(b) Either

$$
h_{2}(v)=v^{-\beta} \quad \text { or } \quad h_{2}(v)=\frac{v^{1-\beta}-(v+s)^{1-\beta}}{(1-\beta) s},
$$

where $s>0$ and $s / Y$ is sufficiently small.

Thus $h_{1}(u)$ is a holomorphic function in $G=\{u \in \mathbb{C}: \operatorname{Re} u \in(X / 2,3 X)\}$ satisfying the approximation (5.2) in $G$; and similarly for $h_{2}(v)$ and $G^{\prime}=$ $\{v \in \mathbb{C}: \operatorname{Re} v \in(Y / 2,3 Y)\}$.

We further suppose that $\mathbf{D}$ is a rectangle.

We can now make some observations useful for verification of the omega conditions, with $f_{1}, \varrho F$ in place of $f, F$. The condition $\left(\Omega_{1}\right)$ gives no difficulty. Interchanging $\alpha, \beta$ if necessary, we suppose that

$$
\left|f_{1}^{(2,0)}(u, v)\right| \gg 1 \quad \text { on } \mathbf{D} \text {. }
$$

Let us define $\psi$ as in (5.4), with $f_{1}$ in place of $f$. Then:

(i) For fixed real $k$ and $l$, the equation

$$
f_{1}^{(1,0)}(k, v)=l
$$

has $O(1)$ solutions $v \in J$.

Take a suitable rectangle $R$ in $G^{\prime}$ containing $J$ in its interior. We readily obtain a holomorphic function $g$ on $G$ such that $|g(v)|<\left|f_{1}^{(1,0)}(k, v)\right|$ on $R$, namely

$$
f_{1}^{(1,0)}(k, v)+g(v)=A k^{-\alpha-1} v^{-\beta} \frac{r}{v} T_{1,0}\left(\frac{q v}{r k}\right)
$$

for $v \in G$. From Rouché's theorem, the equation (5.21) has $O(1)$ solutions inside $R$.

(ii) For fixed real $k$ and $l$, the equation

$$
\psi(l, v)=k
$$

has $O(1)$ solutions $v \in J$.

For if $\psi(l, v)=k$, then $f_{1}^{(1,0)}(k, v)=l$. This equation has $O(1)$ solutions $v \in J$ from (i).

(iii) For fixed real $k^{\prime}$ and $l$, the equation

$$
\frac{\partial \psi}{\partial v}(l, v)=k^{\prime}
$$

has $O(1)$ solutions $v \in J$. 
For (5.22) implies

$$
f_{1}^{(1,1)}(\psi(l, v), v)+k^{\prime} f_{1}^{(2,0)}(\psi(l, v), v)=0 .
$$

In view of (ii), we need only show that

$$
f_{1}^{(1,1)}(k, v)+k^{\prime} f_{1}^{(2,0)}(k, v)=0
$$

has $O(1)$ solutions $v \in J$. This is accompanied by an application of Rouché's theorem much as above.

(iv) Let $q$ be a given function on $\mathbb{R}^{2}$. Suppose that

$$
h(v):=-q^{(1,0)}(k, v) f_{1}^{(1,1)}(k, v)+q^{(0,1)}(k, v) f_{1}^{(2,0)}(k, v)
$$

is holomorphic in $G$ for any fixed $k$ in $I$. Suppose further that $h$ has only $O(1)$ zeros on the interval $J$. Then the equation

$$
(q(\psi(l, v), v))^{\prime}=0
$$

has $O(1)$ solutions $v$ in $J$ for fixed $l$. To see this, we apply Lemma 12 again. Abbreviating $\psi(l, v)$ to $\psi$, we have

$$
\begin{aligned}
(q(\psi(l, v), v))^{\prime} & =q^{(1,0)}(\psi, v) \frac{\partial \psi}{\partial v}+q^{(0,1)}(\psi, v) \\
& =\frac{-q^{(1,0)}(\psi, v) f_{1}^{(1,1)}(\psi, v)+q^{(0,1)}(\psi, v) f_{1}^{(2,0)}(\psi, v)}{f_{1}^{(2,0)}(\psi, v)} .
\end{aligned}
$$

Our claim now follows from observation (ii) and the hypothesis concerning the zeros of $h$.

The domains $\mathbf{D}(q, r, \theta), \mathbf{D}^{\prime}(q, r, \theta)$ take the form

$$
\left\{(u, v) \in \mathbf{D}: H f_{1} \in I^{\prime}\right\}
$$

where $\mathbf{D}$ has the property assumed above and $I^{\prime}$ is an interval. Thus for $\left(\Omega_{2}\right)$, we need to show that $\left(H f_{1}\right)(u, k)$ is $C$-monotonic in $u$ for fixed $k, C=O(1)$. For $\left(\Omega_{3}\right),\left(\Omega_{4}\right)$ we need statements of the form " $q(\psi(l, v), v)$ is $C$-monotonic in $v$ for fixed $l$ " with $C=O(1)$. For $\left(\Omega_{3}\right)$, we must take $q=H f_{1}$, and for $\left(\Omega_{4}\right), q=f_{1}^{(2,0)}$. Thus the verification of these two conditions can be completed by showing (for both choices of $q$ ) that the equation $h(v)=0$ has $O(1)$ solutions $v$ in $J$. As above, all we need is a suitably chosen rectangle $R$, containing $J$ in its interior, and a holomorphic function $g,|g(v)|<|h(v)|$ on $R$, such that $g+h$ is of a simple form and can be seen to have finitely many zeros in $G$. The case $q=H f_{1}$ is distinctly more difficult.

Looking ahead to Theorem 8 , we now take $(\alpha, \beta)=(1,2)$. Routine calculations, using the approximations to $H f_{1}$ and $f_{1}^{(a, b)}$ already found above, give the desired approximation $g(v)+h(v)$ to $h(v)$. No matter what the value of $k$, the rational function $g+h$ cannot vanish identically. This is a matter of examining the roots of certain quadratic and linear polynomials, which 
we leave to the reader. In this way we can verify $\left(\Omega_{3}\right)$. The corresponding tasks for $\left(\Omega_{4}\right)$ and $\left(\Omega_{2}\right)$ are similar but simpler.

We now apply Theorem 7 to Type I sums.

Theorem 8. Let $D \geq 1, M N \asymp D, X \gg N D^{1 / 6}, L=\log (X D+2)$, $\left|a_{n}\right| \leq 1$. Let $I_{m} \subseteq(N, 2 N]$ and

$$
S_{1}=\sum_{m \sim M} a_{m} \sum_{n \in I_{m}} e\left(\frac{X M N}{m n}\right) .
$$

Then

$$
\begin{aligned}
S_{1} \ll & L^{3 / 2}\left\{D^{11 / 12}+D N^{-1 / 2}+X^{-1 / 14} D^{27 / 28} N^{1 / 14}+X^{1 / 8} D^{13 / 16} N^{-1 / 8}\right. \\
& +X^{1 / 16} D^{27 / 32} N^{-1 / 16}+X^{1 / 14} N^{-1 / 7} D^{6 / 7} \\
& \left.+X^{1 / 6} D^{5 / 6} N^{-1 / 6} N_{0}^{-1 / 6}+X^{1 / 26} D^{10 / 13} N^{2 / 13}\right\} .
\end{aligned}
$$

Proof. Let $Q$ be a positive integer, $Q<c N$. Just as in the proof of Theorem 4 , there is a $q \in[1, Q]$ and a rectangle $\mathbf{D} \subseteq[M, 2 M] \times[N, 2 N]$ for which

$$
S_{1}^{2} \ll L D^{2} Q^{-1}+\frac{L^{2} D q}{Q} \sum_{(m, n) \in \mathbf{D}} e\left(X M N m^{-1}\left((n+q)^{-1}-n^{-1}\right)\right) .
$$

Let $N_{0}=\min (M, N)$. We apply Theorem 7 to the sum on the right-hand side of (5.24), replacing $F$ by $X q / N,(X, Y, N)$ by $\left(D / N_{0}, N_{0}, D\right)$ and $\eta$ by $Q / N$. (We note that $X q / N \gg D^{1 / 6}$.) Thus

$$
\begin{aligned}
L^{-3} S_{1}^{2} \ll & D^{2} Q^{-1}+D\left\{(X Q / N)^{1 / 3} D^{1 / 2}+D^{5 / 6}+(X Q / N)^{-1 / 8} D^{15 / 16}\right. \\
& +(Q / N)^{2 / 5} D^{1 / 2}(X Q / N)^{1 / 5} N_{0}^{2 / 5}+(Q / N)^{1 / 4} D^{3 / 4} N_{0}^{1 / 4} \\
& +(Q / N)^{1 / 2}(X Q / N)^{1 / 4} D^{1 / 2} N_{0}^{1 / 4}+D^{1 / 2}(X Q)^{1 / 2} N^{-1 / 2} N_{0}^{-1 / 2} \\
& \left.+D^{1 / 2}(X Q)^{1 / 12} N^{-1 / 12} N_{0}^{5 / 12}\right\} .
\end{aligned}
$$

We simplify this bound using $N_{0} \leq N$. We further restrict $Q$ by

$$
Q<X^{1 / 7} D^{1 / 14} N^{-1 / 7},
$$

so that

$$
(X Q)^{-1 / 8} D^{31 / 16} N^{1 / 8}<D^{2} / Q .
$$

It follows that, for $0<Q<\min \left(c N, X^{1 / 7} D^{1 / 14} N^{-1 / 7}\right)$, we have

$$
\begin{aligned}
L^{-3} S_{1}^{2} \ll & D^{2} Q^{-1}+X^{1 / 3} D^{3 / 2} N^{-1 / 3} Q^{1 / 3}+D^{11 / 6} \\
& +D^{11 / 8} X^{1 / 2} Q^{1 / 2} N^{-1 / 2} N_{0}^{-1 / 4}+X^{1 / 5} D^{3 / 2} N^{-1 / 5} Q^{3 / 5} \\
& +D^{7 / 4} Q^{1 / 4}+X^{1 / 4} D^{3 / 2} N^{-1 / 2} Q^{3 / 4}+D^{3 / 2} X^{1 / 2} Q^{1 / 2} N^{-1 / 2} N_{0}^{-1 / 2} \\
& +D^{3 / 2} X^{1 / 12} Q^{1 / 12} N^{1 / 3} .
\end{aligned}
$$


Applying Lemma 3, we find that

$$
\begin{aligned}
L^{-3} S_{1}^{2} \ll & D^{11 / 6}+X^{1 / 4} D^{13 / 8} N^{-1 / 4}+D^{19 / 12} X^{1 / 3} N^{-1 / 3} N_{0}^{-1 / 6} \\
& +X^{1 / 8} D^{27 / 16} N^{-1 / 8}+D^{2} N^{-1}+D^{27 / 14} X^{-1 / 7} N^{1 / 7}+X^{1 / 7} N^{-2 / 7} D^{12 / 7} \\
& +D^{5 / 3} X^{1 / 3} N^{-1 / 3} N_{0}^{-1 / 3}+D^{20 / 13} X^{1 / 13} N^{4 / 13} .
\end{aligned}
$$

Theorem 8 follows at once.

6. Proof of Theorem 1: initial steps. In this section, let $s=\sigma+i t$ denote a complex variable.

Lemma 18. Assume the Riemann hypothesis, and let $\sigma \in(1 / 2,2]$. For $y \geq 1$, we have

$$
\sum_{n \leq y} \frac{\mu(n)}{n^{s}}=\frac{1}{\zeta(s)}+O\left(y^{1 / 2-\sigma+\varepsilon}\left(|t|^{\varepsilon}+1\right)\right) .
$$

Proof. This is proved in all essentials in [22, §14.25].

We shall write

$$
\begin{aligned}
r(n) & =\sum_{\substack{(u, v) \in \mathbb{Z}^{2} \\
|u|^{3}+|v|^{3}=n}} 1, \quad b=\frac{2 \Gamma^{2}(1 / 3)}{3 \Gamma(2 / 3)}, \\
\Delta(x) & =\sum_{n \leq x} r(n)-b x^{2 / 3}, \quad Z(s)=\sum_{n \geq 1} \frac{r(n)}{n^{s}} \quad(\sigma>1) .
\end{aligned}
$$

Nowak [17] showed (in a more general context) that $Z(s)$ has an analytic continuation to $\sigma>2 / 9$ with the exception of a simple pole at $s=2 / 3$, with residue $2 b / 3$. His discussion yields the estimate

$$
Z(s) \ll|t|^{9 / 7(1-\sigma+\varepsilon)} \quad(\sigma \geq 2 / 9+\varepsilon,|t| \geq 1) .
$$

Lemma 19. Let $\lambda$ be a constant, $2 / 9<\lambda<1 / 2$, and suppose that, for every $\varepsilon>0$,

$$
\int_{T}^{2 T}|Z(\lambda+i t)| d t \ll T^{1+\varepsilon}
$$

for $T \geq 1$. Then for $\varepsilon>0,1 \leq y<x^{1 / 3}$, we have

$$
E(x)=\sum_{d \leq y} \mu(d) \Delta\left(\frac{x}{d^{3}}\right)+O\left(x^{\lambda+\varepsilon} y^{1 / 2-3 \lambda}\right) .
$$


Proof. This is essentially stated in [25]. We give details for the convenience of the reader, following [17]. First of all,

$$
\begin{aligned}
V(x) & =\sum_{|m|^{3}+|n|^{3} \leq x} \sum_{\substack{d \geq 1 \\
d|m, d| n}} \mu(d)=\sum_{\substack{d \geq 1 \\
d^{3}\left(|m|^{3}+|n|^{3}\right) \leq x}} \mu(d) \\
& =\sum_{\substack{d \geq 1, t \geq 1 \\
d^{3} t \leq x}} \mu(d) r(t)=b x^{2 / 3} \sum_{d \leq y} \frac{\mu(d)}{d^{2}}+\sum_{d \leq y} \mu(d) \Delta\left(\frac{x}{d^{3}}\right)+Q(x),
\end{aligned}
$$

where

$$
Q(x)=\sum_{\substack{d>y, t \geq 1 \\ d^{3} t \leq x}} \mu(d) r(t) .
$$

Now let

$$
f(s)=\frac{1}{\zeta(s)}-\sum_{n \leq y} \mu(n) n^{-s} \quad(\sigma>1 / 2),
$$

so that

$$
f(3 s) Z(s)=\sum_{n \geq 1} \frac{a(n)}{n^{s}}, \quad a(n)=\sum_{\substack{d>y, t \geq 1 \\ d^{3} t=n}} \mu(d) r(t) .
$$

An application of Perron's formula gives

$$
Q(x)=\frac{1}{2 \pi i} \int_{2-i x^{C}}^{2+i x^{C}} f(3 s) Z(s) \frac{x^{s}}{s} d s+O(1)
$$

for any constant $C>2$. We move the vertical segment to the left. For a sufficiently large $C$, we obtain

$$
\begin{aligned}
Q(x) & =\frac{1}{2 \pi i} \int_{\lambda-i x^{C}}^{\lambda+i x^{C}} f(3 s) Z(s) \frac{x^{s}}{s} d s+\operatorname{Res}\left(\frac{f(3 s) Z(s) x^{s}}{s}, \frac{2}{3}\right)+O(1) \\
& =\frac{1}{2 \pi i} \int_{\lambda-i x^{C}}^{\lambda+i x^{C}} f(3 s) Z(s) \frac{x^{s}}{s} d s+b x^{2 / 3} \sum_{d>y} \frac{\mu(d)}{d^{2}}+O(1)
\end{aligned}
$$

on applying (6.1) on the horizontal segments.

By a splitting-up argument, there is a $T, 1 \leq T \leq x^{C}$, such that

$$
\begin{aligned}
\left|\int_{\lambda-i x^{C}}^{\lambda+i x^{C}} f(3 x) Z(s) \frac{x^{s}}{s} d s\right| & \ll \frac{x^{\lambda} \log x}{T} \int_{T-1}^{2 T}|f(3 \lambda+3 i t) Z(\lambda+i t)| d t \\
& \ll x^{\lambda+\varepsilon} y^{1 / 2-3 \lambda} .
\end{aligned}
$$


The last estimate follows from Lemma 18 and (6.2). The lemma follows at once on combining (6.3)-(6.5), since

$$
b \sum_{d \geq 1} \frac{\mu(d)}{d^{2}}=\frac{6 b}{\pi^{2}}=\frac{4 \Gamma^{2}(1 / 3)}{\pi^{2} \Gamma(2 / 3)} .
$$

The best available value of $\lambda$ at present is 4/9:

Lemma 20. For $T \geq 1$,

$$
\int_{T}^{2 T}|Z(4 / 9+i t)| d t \ll T \log T .
$$

Proof. This follows from Lemma 3.1 of Zhai [25] on applying the CauchySchwarz inequality.

Let $\psi(t)=\{t\}-1 / 2$, where \{\} denotes the fractional part.

LEMMa 21. We may write

$$
\Delta(x)=\Delta_{1}(x)+\Delta_{2}(x)
$$

where, for a positive constant $c_{1}$,

$$
\begin{aligned}
& \Delta_{1}(x)=c_{1} x^{2 / 9} \sum_{l=1}^{\infty} \frac{1}{l^{4 / 3}} \cos 2 \pi\left(l x^{1 / 3}-1 / 3\right)+O(1), \\
& \Delta_{2}(x)=-8 \sum_{(x / 2)^{1 / 3} \leq n \leq x^{1 / 3}} \psi\left(\left(x-n^{3}\right)^{1 / 3}\right)+O(1) .
\end{aligned}
$$

Proof. See Krätzel [12, Chapter 3].

On combining the last three lemmas, we obtain the decomposition $E(x)$ $=E_{1}(x)+E_{2}(x)+E_{3}(x)$ where (for a parameter $y$ in $\left[1, x^{1 / 3}\right]$ which is at our disposal)

$$
\begin{aligned}
& E_{1}(x)=c_{1} x^{2 / 9} \sum_{d \leq y} \frac{\mu(d)}{d^{2 / 3}} \sum_{k=1}^{\infty} \frac{1}{k^{4 / 3}} \cos 2 \pi\left(\frac{k x^{1 / 3}}{d}-\frac{1}{3}\right), \\
& E_{2}(x)=-8 \sum_{d \leq y} \mu(d) \sum_{x^{1 / 3} /\left(2^{1 / 3} d\right) \leq n \leq x^{1 / 3} / d} \psi\left(\left(\frac{x}{d^{3}}-n^{3}\right)^{1 / 3}\right), \\
& E_{3}(x)=O\left(x^{4 / 9+\varepsilon} y^{-5 / 6}+y\right) .
\end{aligned}
$$

To obtain (1.2), we choose $y=x^{8 / 15-6 \theta / 5}$. It now suffices to show, for any $D, D^{\prime}$ with $1 \leq D \leq y, D<D^{\prime} \leq 2 D$, and any $K \geq 1$, that

$$
\sum_{k \sim K} \sum_{D \leq d \leq D^{\prime}} \mu(d) e\left(\frac{k x^{1 / 3}}{d}\right) \ll K^{4 / 3} D^{2 / 3} x^{\theta-2 / 9+\varepsilon}
$$


and

$$
\sum_{d \sim D} \mu(d) \sum_{n \sim x^{1 / 3} /\left(2^{1 / 3} d\right)} \psi\left(\left(\frac{x}{d^{3}}-n^{3}\right)^{1 / 3}\right) \ll x^{\theta+\varepsilon} .
$$

We complete this section with a proof of a stronger result than (6.7), namely

$$
\begin{aligned}
S(D) & :=\sum_{d \sim D} \mu(d) \sum_{n \sim x^{1 / 3} /\left(2^{1 / 3} d\right)} \psi\left(\left(\frac{x}{d^{3}}-n^{3}\right)^{1 / 3}\right) \\
& \ll x^{7 / 27+\varepsilon} \quad\left(D \ll x^{2 / 9}\right) .
\end{aligned}
$$

(This is the bound corresponding to (6.7) if $\theta$ is replaced by $7 / 27$.)

Lemma 22. For $H \geq 1$, we have a representation

$$
\psi(u)=\sum_{1 \leq|h| \leq H} a(h) e(h u)+O\left(\sum_{1 \leq h \leq H} b(h) e(h u)\right)+O\left(H^{-1}\right)
$$

with coefficients $a(h) \ll 1 /|h|, b(h) \ll 1 / H$.

Proof. See Vaaler [23], or the appendix to [6].

We now split up $S(D)$ as follows. For $d \sim D$ (suppressing dependence on $d$ ), let

$$
N_{j}=\frac{x^{1 / 3}}{d\left(1+2^{-3 j / 2}\right)^{1 / 3}}, \quad j=0,1, \ldots, J .
$$

Here $J$ is the least integer such that $x^{1 / 3} / d-N_{J} \leq x^{\varepsilon}$. Thus $J \ll \log x$, $N_{j+1}-N_{j} \asymp x^{1 / 3} 2^{-3 j / 2} D^{-1} \gg x^{\varepsilon}$, and in particular

$$
x^{1 / 3} 2^{-3 j / 2} D^{-1} \gg x^{\varepsilon}
$$

for $j=0,1, \ldots, J$.

It suffices to show that for each $j=0, \ldots, J$,

$$
\sum_{d \sim D} \mu(d) \sum_{n \in I_{d}} \psi\left(\left(\frac{x}{d^{3}}-n^{3}\right)^{1 / 3}\right) \ll x^{7 / 27+\varepsilon},
$$

where $I_{d}=I_{d}(j)=\left[N_{j}, N_{j+1}\right]$.

It is convenient to write $P$ for $2^{j}$. We apply Lemma 22 with

$$
H=\max \left(x^{2 / 27} P^{-3 / 2}, 1\right) \text {. }
$$


Thus the sum in (6.10) can be rewritten as

$$
\begin{aligned}
\sum_{d \sim D} \mu(d) & \sum_{1 \leq|h| \leq H} a(h) \sum_{n \in I_{d}} e\left(h\left(\frac{x}{d^{3}}-n^{3}\right)^{1 / 3}\right) \\
& +O\left(\sum_{d \sim D} \sum_{1 \leq h \leq H} b(h) \sum_{n \in I_{d}} e\left(h\left(\frac{x}{d^{3}}-n^{3}\right)^{1 / 3}\right)\right)+O\left(x^{7 / 27}\right) .
\end{aligned}
$$

We need only show that, for $1 \leq K \leq H$ and $\left|a_{h}\right| \leq 1$,

$$
\begin{aligned}
S(D, K, P) & :=K^{-1} \sum_{\substack{d \sim D \\
h \sim K}} \mu(d) \sum_{h \sim I_{h}} \sum_{n \in I_{d}} e\left(h\left(\frac{x}{d^{3}}-n^{3}\right)^{1 / 3}\right) \\
& \ll x^{7 / 27+\varepsilon} .
\end{aligned}
$$

The corresponding result with 1 in place of $\mu(d)$ is, of course, easier.

We apply the $B$-process to the sum over $n$ in (6.10). We may quote the result from Kühleitner [13, (3.5)]:

$$
S(D, K, P) \ll \frac{x^{1 / 6}}{P^{5 / 4} D^{1 / 2} K^{3 / 2}}\left|S^{\prime}(D, K, P)\right|+D \log x,
$$

with

$$
S^{\prime}(D, K, P)=\sum_{d \sim D} \mu(d)\left(\frac{D}{d}\right)^{1 / 2} \sum_{(h, m) \in \mathbf{T}} b(h, m) e\left(\frac{-x^{1 / 3}|(h, m)|_{3 / 2}}{d}\right) .
$$

Here $b(h, m) \ll 1$ and $\mathbf{T}=\{(h, m): h \sim K, P h \leq m \leq 2 P h\}$. Thus we must show that

$$
\begin{aligned}
\sum_{D<d \leq D^{\prime}} \mu(d) \sum_{(h, m) \in \mathbf{T}} b(h, m) e\left(\frac{-x^{1 / 3}|(h, m)|_{3 / 2}}{d}\right) \\
\ll x^{5 / 54+\varepsilon} P^{5 / 4} D^{1 / 2} K^{3 / 2} .
\end{aligned}
$$

If $K<x^{5 / 27} P^{1 / 2} D^{-1}$, then (6.14) is trivial. We now assume that

$$
H \geq K \geq x^{5 / 27} P^{1 / 2} D^{-1} \text {. }
$$

We next dispose of the case where

$$
K<\min \left(x^{-2 / 27} P^{-1 / 4} D^{1 / 2}, x^{5 / 27} P^{1 / 2} D^{-2 / 3}\right)
$$

by treating the variables $h, m$ trivially in (6.14). In view of Lemma 2(ii), we need only show that, for $\left|a_{m}\right| \leq 1,\left|b_{n}\right| \leq 1$,

$$
\sum_{\substack{m \sim M, n \sim N \\ D<m n \leq D^{\prime}}} a_{m} b_{n} e\left(\frac{Y}{m n}\right) \ll x^{5 / 54+\varepsilon} P^{1 / 4} D^{1 / 2} K^{-1 / 2}
$$

whenever

$$
D^{1 / 3} \ll N \ll D^{1 / 2}, \quad Y \asymp x^{1 / 3} P K,
$$


and that for $\left|a_{m}\right| \leq 1$,

$$
\sum_{\substack{m \sim M, n \sim N \\ D<m n \leq D^{\prime}}} a_{m} e\left(\frac{Y}{m n}\right) \ll x^{5 / 54+\varepsilon} P^{1 / 4} D^{1 / 2} K^{-1 / 2}
$$

whenever

$$
N \gg D^{2 / 3}, \quad Y \asymp x^{1 / 3} P K
$$

For (6.17), we use Lemma 5 with $M_{1}=M, M_{2}=1, M_{3}=N, M_{4}=1$. The left-hand side of $(6.17)$ is

$$
\begin{aligned}
& \ll(\log x)^{2}\left\{Y^{1 / 2}+M^{1 / 2} N+M N^{1 / 2}+M N(Y / D)^{-1 / 2}\right\} \\
& \ll(\log x)^{2}\left\{x^{1 / 6} P^{1 / 2} K^{1 / 2}+D^{5 / 6}+D^{3 / 2} x^{-1 / 6} P^{-1 / 2} K^{-1 / 2}\right\} \\
& \ll(\log x)^{2}\left\{x^{1 / 6} P^{1 / 2} K^{1 / 2}+D^{5 / 6}\right\} \ll x^{5 / 54+\varepsilon} P^{1 / 4} D^{1 / 2} K^{-1 / 2},
\end{aligned}
$$

where we appeal to (6.16) in the last step.

For (6.19), we treat $m$ trivially and estimate the sum over $n$ using the exponent pair $(1 / 2,1 / 2)$. The left-hand side of $(6.19)$ is

$\ll M\left(\frac{Y}{D}\right)^{1 / 2}+M\left(\frac{Y}{D N}\right)^{-1} \ll M D^{-1 / 2} x^{1 / 6} P^{1 / 2} K^{1 / 2}+D^{2} x^{-1 / 3} P^{-1} K^{-1}$.

Certainly $D^{2} x^{-1 / 3} P^{-1} K^{-1} \ll x^{5 / 54} P^{1 / 4} D^{1 / 2} K^{-1 / 2}$, and we obtain

$$
M D^{-1 / 2} x^{1 / 6} P^{1 / 2} K^{1 / 2} \ll x^{5 / 54} P^{1 / 4} D^{1 / 2} K^{-1 / 2}
$$

by appealing to (6.16) and (6.18). This completes the treatment of the case (6.16).

We note in particular that (6.14) holds whenever $H=1$, for in this case $K=1$, while (6.15) gives $D \geq x^{5 / 27} P^{1 / 2}$. Now (6.16) is easily verified. We may now suppose that $H=x^{2} / 27 P^{-3 / 2}$. Since $K \leq H$, we have

$$
K P^{3 / 2} \leq x^{2 / 27}, \quad P \leq x^{4 / 81} .
$$

We are now in a position to apply Theorem 2 with $(\kappa, \lambda)=(1 / 2,1 / 2)$ and essentially $\left(D, K, P K, x^{1 / 3} P K D^{-1}\right)$ in place of $\left(M, M_{1}, M_{2}, X\right)$. We may suppose, in addition to (6.21), that

$$
K \geq D P^{-1 / 2} x^{-5 / 27},
$$

for in the contrary case we note that (6.16) holds, since

$$
\begin{gathered}
D P^{-1 / 2} x^{-5 / 27}\left(x^{-2 / 27} P^{-1 / 4} D^{1 / 2}\right)^{-1} \ll D^{1 / 2} x^{-3 / 27} \ll 1, \\
D P^{-1 / 2} x^{-5 / 27}\left(x^{5 / 27} P^{1 / 2} D^{-2 / 3}\right)^{-1} \ll D^{5 / 3} x^{-10 / 27} \ll 1 .
\end{gathered}
$$

The condition $X \gg M_{2}^{2}$ in Theorem 2 reduces to $x^{1 / 3} P K D^{-1} \gg P^{2} K^{2}$, that is, $D P K \ll x^{1 / 3}$. This is a consequence of (6.21). Thus the left-hand 
side of $(6.14)$ is

$$
\begin{aligned}
& \ll x^{\varepsilon}\left(D P K+D^{2 / 3}(P K)^{2}\left(x^{1 / 3} /(D P K)\right)^{1 / 6}\right) \\
& \ll x^{\varepsilon} D P K+D^{1 / 2} x^{1 / 18+\varepsilon}(P K)^{11 / 6} .
\end{aligned}
$$

It remains to show that

$$
D P K \leq x^{5 / 54} P^{5 / 4} D^{1 / 2} K^{3 / 2}
$$

(which is simply (6.22)), and that

$$
D^{1 / 2} x^{1 / 18}(P K)^{11 / 6} \ll x^{5 / 54} P^{5 / 4} D^{1 / 2} K^{3 / 2},
$$

that is, $P^{7 / 12} K^{1 / 3} \ll x^{1 / 27}$. This is an easy consequence of $(6.21)$ :

$$
P^{7 / 12} K^{1 / 3}=P^{1 / 12}\left(P^{3 / 2} K\right)^{1 / 3} \ll x^{1 / 243+2 / 81} .
$$

This completes the proof of (6.14).

7. Completion of the proof of Theorem 1. It remains to prove (6.6). We write $D=x^{\phi}$. Since the trivial bound gives (6.6) for $\phi \leq 3 \theta-2 / 3$, we assume that

$$
0.113 \ldots=3 \theta-\frac{2}{3}<\phi \leq \frac{8}{15}-\frac{6 \theta}{5}=0.221 \ldots
$$

We fix $K \geq 1$ and $D^{\prime}, D \leq D^{\prime}<2 D$. Let

$$
S_{1}=\sum_{l \sim K} \sum_{\substack{m \sim M \\ D \leq m n<D^{\prime}}} \sum_{\substack{n \sim N \\ D \leq m}} a_{m} e\left(\frac{l x^{1 / 3}}{m n}\right), \quad S_{2}=\sum_{l \sim K} \sum_{\substack{m \sim M \\ D \leq m n<D^{\prime}}} \sum_{\substack{n \sim N \\ D \leq m}} a_{m} b_{n} e\left(\frac{l x^{1 / 3}}{m n}\right)
$$

with coefficients satisfying $\left|a_{m}\right| \leq 1,\left|b_{n}\right| \leq 1$.

LEMMA 23. Suppose that

$$
N \gg D^{2 / 3} x^{4 / 9-2 \theta} .
$$

Then

$$
S_{1} \ll K^{4 / 3} D^{2 / 3} x^{\theta-2 / 9+\varepsilon}
$$

provided that either

$$
N \gg D^{-25 / 21} x^{-50 \theta / 7+19 / 9}
$$

or

$$
N \gg D^{-944 / 267} x^{-1888 \theta / 89+4898 / 801} .
$$

Proof. It suffices to show that

$$
S_{1}^{\prime}:=\sum_{\substack{m \sim M \\ D \leq m n<D^{\prime}}} \sum_{\substack{n \sim N \\ D \leq m}} a_{m} e\left(\frac{l x^{1 / 3}}{m n}\right) \ll l^{1 / 3} D^{2 / 3} x^{\theta-2 / 9+\varepsilon} .
$$


We appeal to Theorem 4 with $X \asymp l x^{1 / 3} D^{-1}$. In (4.15), the terms $D N^{-1 / 2}$, $D X^{-1 / 2}$ are acceptable because of $(7.2)$. As $N_{0} \leq N$ and $(1+2 \kappa) /(6+4 \kappa)$ $\leq 1 / 4$, we need only show that

$$
\left(D^{4+4 \kappa}\left(x^{1 / 3} D^{-1}\right)^{1+2 \kappa} N^{-(1+4 \kappa-2 \lambda)}\right)^{1 /(6+4 \kappa)} \ll D^{2 / 3} x^{\theta-2 / 9} .
$$

The condition (7.4) arises on choosing $(\kappa, \lambda)=(2 / 7,4 / 7)=B A^{2} B(0,1)$, while $(7.5)$ arises from $(\kappa, \lambda)=(89 / 570,1 / 2+89 / 570)$. The latter exponent pair requires lengthy arguments (Huxley [9, Chapter 17]).

We remark that the slightly stronger exponent pair in [10], $(32 / 205,1 / 2+$ $32 / 205)$, would not significantly "reduce $\theta$ ".

Lemma 24. Suppose that (7.2) holds, and that $\phi \leq 4(\theta-2 / 9)=0.151 \ldots$,

$$
N \leq D^{-25 / 21} x^{-50 \theta / 7+19 / 9},
$$

and

$$
N \geq x^{19 / 9-8 \theta} D^{1 / 6}
$$

Then (7.3) holds.

Proof. It suffices to prove (7.6). Since $N \ll D \ll x^{1 / 3} D^{-7 / 6}$, we may appeal to Theorem 8 with $X \asymp l x^{1 / 3} D^{-1}$. Thus

$$
\begin{aligned}
S_{1} \ll & (\log x)^{3 / 2} l^{1 / 6}\left\{D^{11 / 12}+D N^{-1 / 2}+x^{-1 / 42} D^{29 / 28} N^{1 / 14}\right. \\
& +x^{1 / 24} D^{11 / 16} N^{-1 / 8}+x^{1 / 48} D^{25 / 32} N^{-1 / 16}+x^{1 / 42} D^{11 / 14} N^{-1 / 7} \\
& \left.+D^{2 / 3} x^{1 / 18} N^{-1 / 3}+x^{1 / 18} D^{1 / 2}+x^{1 / 78} D^{19 / 26} N^{2 / 13}\right\} .
\end{aligned}
$$

The first term on the right-hand side of (7.9) is acceptable as $\phi \leq 4(\theta-2 / 9)$. The second term is acceptable because of (7.2). The third term is acceptable because of (7.7), which is stronger than the required condition

$$
N \leq D^{-31 / 6} x^{14 \theta-25 / 9}
$$

since $\phi<0.152$.

The fourth, fifth, sixth and seventh terms are acceptable because of (7.8). The eighth term is acceptable since $D>x^{0.11}$. The last term is also acceptable because of (7.7). This completes the proof of the lemma.

Lemma 25. Let $\phi<1 / 6$. We have

$$
S_{2} \ll K^{4 / 3} D^{2 / 3} x^{\theta-2 / 9+\varepsilon}
$$

provided that (7.2) holds, and either

$$
N \ll \min \left(x^{276 \theta / 5-14} D^{-2}, D^{1 / 2}\right)
$$

or

$$
N \ll \min \left(D^{-2 / 3} x^{1508 \theta / 95-226 / 57}, D^{1 / 2}\right)
$$


Proof. It suffices to show that

$$
S_{2}^{\prime}:=\sum_{\substack{m \sim M \\ D \leq m n<D^{\prime}}} \sum_{\substack{n \sim N \\ D \leq m}} a_{m} b_{n} e\left(\frac{l x^{1 / 3}}{m n}\right) \ll l^{1 / 3} D^{2 / 3} x^{\theta-2 / 9+\varepsilon} .
$$

We appeal to Theorem 5 with $X \asymp l x^{1 / 3} / D \gg D$. Again, $D N^{-1 / 2}$ is acceptable. Since $M \gg D^{1 / 2}$ and $\phi<1 / 6$, we have

$$
D M^{-1 / 4}\left(D^{2 / 3} x^{\theta-2 / 9}\right)^{-1} \ll D^{5 / 24} x^{-\theta+2 / 9} \ll 1,
$$

so the term $D M^{-1 / 4}$ is acceptable. Since $(1+2 \kappa) /(14+12 \kappa)<1 / 3$, it remains to show that

$$
\left(D^{11+10 \kappa}\left(x^{1 / 3} / D\right)^{1+2 \kappa} N^{2(\lambda-\kappa)}\right)^{1 /(14+12 \kappa)} \ll D^{2 / 3} x^{\theta-2 / 9} .
$$

The condition (7.11) arises on choosing $(\kappa, \lambda)=(11 / 30,16 / 30)=B A^{3} B(0,1)$, while $(7.12)$ arises from $(\kappa, \lambda)=(89 / 570,1 / 2+89 / 570)$.

Lemma 26. We have (7.10) provided that (7.2) holds and either

$$
\phi<26 / 15-6 \theta=0.17 \ldots, \quad N \ll D^{13 / 6} x^{10 \theta-26 / 9},
$$

or

$$
\phi \geq 26 / 15-6 \theta, \quad N \ll D^{1 / 2} .
$$

Proof. Again, we need only prove (7.13). Lemma 5 with $(\kappa, \lambda)=(1 / 2,1 / 2)$ yields

$$
\begin{aligned}
S_{2}^{\prime} \ll & (\log x)^{3} l^{1 / 5}\left\{x^{1 / 15} D^{9 / 20} N^{1 / 10}+x^{1 / 18} D^{1 / 2} N^{1 / 9}+x^{1 / 15} D^{2 / 5} N^{1 / 5}\right. \\
& +x^{1 / 33} D^{6 / 11} N^{3 / 11}+D^{2 / 3} N^{5 / 18} \\
& \left.+D N^{-1 / 2}+x^{-1 / 66} D^{15 / 22} N^{9 / 22}+D^{3 / 2} x^{-1 / 6}\right\} .
\end{aligned}
$$

The last four terms are easily seen to be acceptable in view of (7.2). Since $N \ll D^{1 / 2}$, we have

$$
\begin{aligned}
x^{1 / 15} D^{2 / 5} N^{1 / 5} & \ll x^{1 / 15} D^{9 / 20} N^{1 / 10}, \\
x^{1 / 33} D^{6 / 11} N^{3 / 11} & \ll x^{1 / 33} D^{15 / 22} \ll D^{2 / 3} x^{\theta-2 / 9} .
\end{aligned}
$$

Moreover,

$$
x^{1 / 15} D^{9 / 20} N^{1 / 10} \ll D^{2 / 3} x^{\theta-2 / 9}
$$

for $N \ll D^{13 / 6} x^{10 \theta-26 / 9}$, and certainly if (7.15) holds.

The remaining term $x^{1 / 18} D^{1 / 2} N^{1 / 9}$ is $\ll D^{2 / 3} x^{\theta-2 / 9}$ for $N \ll D^{3 / 2} x^{9 \theta-5 / 2}$, which holds if either (7.14) or (7.15) is assumed.

Lemma 27. We have (7.10) provided that

$$
N \gg D^{4 / 3} x^{8 / 9-4 \theta},
$$

and either

$$
\phi<22 / 21-24 \theta / 7=0.155 \ldots, \quad N \ll D^{5 / 3} x^{4 \theta-11 / 9},
$$


or

$$
\phi \geq 22 / 21-24 \theta / 7, \quad N \ll D^{1 / 2} .
$$

Proof. From Lemma 7 , essentially with $\left(K, N, M, K x^{1 / 3} / D\right)$ in place of $(H, N, M, X)$, we have

$$
S_{2} \ll x^{\varepsilon} K\left\{x^{1 / 12} D^{1 / 4} N^{1 / 4}+D N^{-1 / 4}+D^{1 / 2} N^{1 / 2}+D^{3 / 2} x^{-1 / 6}\right\} .
$$

The last term has already been discussed above. The second term is acceptable since (7.16) holds. The first term is acceptable since (7.17) (or, if $\phi \geq 22 / 21-24 \theta / 7$, the stronger condition (7.18)) holds. Finally, the third term is acceptable since $N \ll D^{1 / 2}$.

LEMMA 28. Let

$$
\phi<\frac{24}{5}\left(\theta-\frac{2}{9}\right)=0.18 \ldots
$$

We have (7.10) provided that (7.2) holds and

$$
N \ll \min \left(\left(D^{659} x^{5076 \theta-1410}\right)^{1 / 187}, D^{1 / 2}\right) .
$$

Proof. We apply Theorem 6 with $\alpha=\beta=-1, X \asymp x^{1 / 3} D^{-1}$, taking $(\kappa, \lambda)=B A(89 / 570,1 / 2+89 / 570)=(187 / 659,374 / 659)$. The term $L^{2} D N^{-1 / 2}$ is acceptable because of (7.2). The term $L^{2} D N^{-1 / 4}$ is acceptable since

$$
D M^{-1 / 4} \ll D^{7 / 8} \ll D^{2 / 3} x^{\theta-2 / 9}
$$

from (7.19). Finally, the term

$$
X^{1 / 6}\left(D^{4+5 \kappa} N^{\lambda-\kappa}\right)^{1 /(6+6 \kappa)} \asymp x^{1 / 18}\left(D^{2725} N^{187}\right)^{1 / 5076}
$$

is acceptable because of (7.20).

Completion of the proof of Theorem 1. We assume (7.1) and show that (6.6) holds.

Suppose first that $\phi>26 / 15-6 \theta$. By Lemma 25, we have (7.13) for

$$
D^{2 / 3} x^{4 / 9-2 \theta} \ll N \ll D^{1 / 2}
$$

and moreover, $D^{2 / 3} x^{4 / 9-2 \theta} \ll D^{1 / 3}$.

By Lemma 23, we have (7.3) for

$$
N \gg D^{-25 / 21} x^{-50 \theta / 7+19 / 9} \text {. }
$$

We note that

$$
D^{-25 / 21} x^{-50 \theta / 7+19 / 9} \ll D^{3 / 5} \text { for } \phi>0.142 .
$$

Now (6.6) follows from Lemma 2(ii).

Suppose next that

$$
0.161 \ldots=\frac{30}{7}\left(\theta-\frac{2}{9}\right)<\phi \leq \frac{26}{15}-6 \theta .
$$


We claim that (7.10) holds for $D^{1 / 4} \ll N \ll D^{2 / 5}$ and (7.3) holds for $N \gg D^{2 / 5}$. This is sufficient for (6.6) in view of Lemma 2(i) with $h=4$.

For $S_{2}$, we use Lemma 28. We have

$$
D^{2 / 3} x^{4 / 9-2 \theta}<D^{1 / 4}
$$

since $\phi \leq 26 / 15-6 \theta<(24 / 5)(\theta-2 / 9)$. We also have

$$
\left(D^{659} x^{5076 \theta-1410}\right)^{1 / 187}>D^{2 / 5}
$$

this requires only $\phi>0.156$. Moreover,

$$
\min \left(D^{1 / 2},\left(D^{659} x^{5076 \theta-1410}\right)^{1 / 187}\right)>D^{-25 / 21} x^{-50 \theta / 7+19 / 9}
$$

this requires only $\phi>0.157$. In view of Lemma 25 , we conclude that (7.13) holds for $D^{1 / 4} \ll N \ll D^{2 / 5}$ and (7.3) holds for $N \gg D^{2 / 5}$, as required for the range (7.22).

Suppose next that

$$
0.156 \ldots=\frac{22}{21}-\frac{24 \theta}{7}<\phi \leq \frac{30}{7}\left(\theta-\frac{2}{9}\right) .
$$

We claim that (7.10) holds for $D^{1 / 5} \ll N \ll D^{1 / 3}$ and $D^{2 / 5} \ll N \ll D^{1 / 2}$, while (7.3) holds for $N \gg D^{2 / 5}$. This is sufficient for (6.6), in view of Lemma 2(iii).

We have

$$
D^{2 / 3} x^{4 / 9-2 \theta} \leq D^{1 / 5}
$$

since $\phi \leq(30 / 7)(\theta-2 / 9)$, while

$$
\left(D^{659} x^{5076 \theta-1410}\right)^{1 / 187}>D^{1 / 3}
$$

this requires only $\phi>0.153$. Thus Lemma 28 gives (7.10) for $D^{1 / 5} \ll N \ll$ $D^{1 / 3}$. Moreover, Lemma 27 gives (7.10) for $D^{2 / 5} \ll N \ll D^{1 / 2}$, and indeed for $D^{2 / 5} \ll N \ll D^{3 / 5}$. Now, recalling (7.21), we have (7.3) for $N \gg D^{2 / 5}$, and we have established (6.6) in the range (7.23).

Suppose next that

$$
0.150 \ldots=4\left(\theta-\frac{2}{9}\right)<\phi \leq \frac{22}{21}-\frac{24 \theta}{7} .
$$

We now use Lemma 25. This yields (7.10) for

$$
D^{2 / 3} x^{4 / 9-2 \theta} \ll N \ll D^{-2 / 3} x^{1508 \theta / 95-226 / 57},
$$

while Lemma 27 yields (7.10) for

$$
D^{4 / 3} x^{8 / 9-4 \theta} \ll N \ll D^{5 / 3} x^{4 \theta-11 / 9} .
$$

Note that

$$
D^{5 / 3} x^{4 \theta-11 / 9} \geq D^{-944 / 267} x^{-1888 \theta / 89+4898 / 801},
$$


since

$$
\phi\left(\frac{5}{3}+\frac{944}{267}\right) \geq 4\left(\theta-\frac{2}{9}\right)\left(\frac{5}{3}+\frac{944}{267}\right)=\frac{4898}{801}+\frac{11}{9}-\theta\left(\frac{1888}{89}+4\right) .
$$

In view of Lemma 23 , we have (7.10) for $N \gg D^{4 / 3} x^{8 / 9-4 \theta}$. We now apply Lemma 2(iv) with

$$
D^{\chi}=D^{2 / 3} x^{4 / 9-2 \theta}, \quad D^{\psi}=D^{-2 / 3} x^{1508 \theta / 95-226 / 57} .
$$

Since $4(\theta-2 / 9)<\phi \leq(30 / 7)(\theta-2 / 9)$, we have $1 / 6<\chi \leq 1 / 5$, and

$$
\max \left(\frac{1}{3}, \frac{1}{5}+\frac{4 \chi}{5}\right)=\frac{1}{5}+\frac{4 \chi}{5} .
$$

Moreover,

$$
D^{-2 / 3} x^{1508 \theta / 95-226 / 57}>D^{1 / 5}\left(D^{2 / 3} x^{4 / 9-2 \theta}\right)^{4 / 5}
$$

this requires only $\phi<0.157$. Thus Lemma 2(iv) is applicable, and (6.6) holds in the range (7.24).

Suppose now that

$$
0.138 \ldots=\frac{2}{5}\left(\frac{276 \theta}{5}-14\right)<\phi \leq 4\left(\theta-\frac{2}{9}\right) .
$$

Then (7.3) holds for

$$
N \gg x^{19 / 9-8 \theta} D^{1 / 6}
$$

To see this, we appeal to Lemma 24. We may suppose that (7.7) holds, in view of Lemma 23.

In order to apply Lemma 2 with $h=5$, we need only verify that

$$
\max \left(D^{1 / 3}, x^{19 / 9-8 \theta} D^{1 / 6}\right)<D^{-2 / 3} x^{1508 \theta / 95-226 / 57} .
$$

This requires only $\phi<0.154$, and we have established (6.6) in the range (7.25).

Suppose finally that

$$
\phi \leq \frac{2}{5}\left(\frac{276 \theta}{5}-14\right) .
$$

From Lemma 25, (7.10) holds for $D^{2 / 3} x^{4 / 9-2 \theta} \ll N \ll D^{1 / 2}$. Moreover, $D^{1 / 3} x^{2 \theta-4 / 9}>D^{2 / 3}$. In view of Lemma 2(ii), it suffices to establish (7.6) for

$$
N>D^{1 / 3} x^{2 \theta-4 / 9} .
$$

We estimate the sum over $m$ in (7.6) trivially and apply the exponent pair $(1 / 6,2 / 3)=A B(0,1)$ to the sum over $n$. Since $l x^{1 / 3} / D>D \gg N$, this gives

$$
S_{1}^{\prime} \ll M\left(\frac{l x^{1 / 3}}{D}\right)^{1 / 6} N^{1 / 2} \ll l^{1 / 6} x^{1 / 18} D^{5 / 6} N^{-1 / 2} \ll l^{1 / 6} D^{2 / 3} x^{\theta-2 / 9}
$$


for $N \gg D^{1 / 3} x^{5 / 9-2 \theta}$. This is stronger than we need, and we have (6.6) for the range (7.27). This completes the proof of Theorem 1.

\section{References}

[1] R. C. Baker, The square-free divisor problem, Quart. J. Math. Oxford 45 (1994), 269-277.

[2] R. C. Baker, G. Harman and J. Rivat, Primes of the form $\left[n^{c}\right]$, J. Number Theory 50 (1995), 261-277.

[3] R. C. Baker and G. Kolesnik, On the distribution of $p^{\alpha}$ modulo one, J. Reine Angew. Math. 356 (1985), 174-193.

[4] E. Bombieri and H. Iwaniec, On the order of $\zeta(1 / 2+i t)$, Ann. Scuola Norm. Sup. Pisa 13 (1986), 449-472.

[5] E. Fouvry and H. Iwaniec, Exponential sums with monomials, J. Number Theory 33 (1989), 311-333.

[6] S. W. Graham and G. Kolesnik, Van der Corput's Method of Exponential Sums, Cambridge Univ. Press, 1991.

[7] D. R. Heath-Brown, Prime numbers in short intervals and a generalized Vaughan identity, Canad. J. Math. 34 (1982), 1365-1377.

[8] —, The Pjateckii-Sapiro prime number theorem, J. Number Theory 16 (1983), 242266.

[9] M. N. Huxley, Area, Lattice Points and Exponential Sums, Oxford Univ. Press, 1996.

[10] - Exponential sums and the Riemann zeta function $V$, Proc. London Math. Soc. (3) 90 (2005), 1-41.

[11] C.-H. Jia, The distribution of square-free numbers, Sci. China Ser. A 36 (1993), 154-169.

[12] E. Krätzel, Lattice Points, Deutscher Verlag Wiss., 1988.

[13] M. Kühleitner, On sums of two kth powers: an asymptotic formula for the mean square of the error term, Acta Arith. 92 (2000), 263-276.

[14] H. L. Montgomery and R. C. Vaughan, The distribution of squarefree numbers, in: Recent Progress in Analytic Number Theory (Durham, 1979), Vol. 1, Academic Press, 1981, 247-256.

[15] B. Z. Moroz, On the number of primitive lattice points in plane domains, Monatsh. Math. 99 (1985), 37-43.

[16] W. Müller and W. G. Nowak, Lattice points in planar domains: applications of Huxley's "discrete Hardy-Littlewood method", in: Number-Theoretic Analysis (Vienna, 1988-1989), Springer, 1990, 139-164.

[17] W. G. Nowak, On sums of two coprime $k$-th powers, Monatsh. Math. 108 (1989), $47-57$.

[18] - On sums and differences of two relative prime cubes, Analysis 15 (1995), 325341.

[19] —, Primitive lattice points in starlike planar sets, Pacific J. Math. 170 (1997), 163-178.

[20] —, On sums and differences of two relative prime cubes II, Tatra Mt. Math. Publ. 11 (1997), 23-34.

[21] O. Robert and P. Sargos, Three-dimensional exponential sums with monomials, J. Reine Angew. Math. 591 (2006), 1-20. 
[22] E. C. Titchmarsh, The Theory of the Riemann Zeta Function, 2nd ed., Oxford Univ. Press, 1986.

[23] J. D. Vaaler, Some extremal problems in Fourier analysis, Bull. Amer. Math. Soc. 12 (1985), 183-216.

[24] J. Wu, On the average number of unitary factors of finite abelian groups, Acta Arith. 84 (1998), 17-29.

[25] W. Zhai, On sums of two coprime $k$-th powers, Monatsh. Math. 144 (2005), 233-250.

[26] W. Zhai and X. Cao, On sums of two coprime $k$-th powers (II), ibid. 148 (2006), $153-172$.

Department of Mathematics

Brigham Young University

Provo, UT 84602, U.S.A.

E-mail: baker@math.byu.edu

Received on 4.8.2006

and in revised form on 24.5.2007 